Edmundo Arteaga-Fernández

\title{
Cardiodesfibrilador implantável na prevenção de morte súbita na cardiomiopatia hipertrófica
}

Tese apresentada à Faculdade de Medicina da Universidade de São Paulo para obtenção do título de Professor Livre-Docente junto ao Departamento de Cardio-Pneumologia (Disciplina de Cardiologia)

São Paulo 
Dados Internacionais de Catalogação na Publicação (CIP)

Preparada pela Biblioteca da

Faculdade de Medicina da Universidade de São Paulo

Creprodução autorizada pelo autor

Arteaga-Fernández, Edmundo

Cardiodesfibrilador implantável na prevenção de morte súbita na cardimiopatia hipertrófica / Edmundo Arteaga-Fernández. -- São Paulo, 2011.

Tese(livre-docência)--Faculdade de Medicina da Universidade de São Paulo. Departamento de Cardio-Pneumologia. Disciplina de Cardiologia.

Descritores: 1.Cardiomiopatia hipertrófica 2.Desfibriladores implantáveis 3.Morte súbita cardíaca 4.Fatores de risco 5.Prevenção primária 6.Prevenção secundária 7.Sobrevida

USP/FM/DBD-112/11 
Dedico esta tese à minha companheira de caminhada Silvia Vaz Domingues e aos nossos filhos universitários: Marcos Mallet Arteaga, Luiza Vaz Domingues Moreno e Beatriz Vaz Domingues Moreno 


\section{Agradecimentos}

Ao Prof. Dr. Charles Mady, o maior e persistente incentivador para a realização da Livre-Docência.

Ao Prof. Dr. José Antonio Franchini Ramires, pela oportunidade de participar deste concurso.

Aos meus colaboradores diretos no estudo da cardiomiopatia hipertrófica: Paulo de Tarso Jorge Medeiros, Murillo Antunes de Oliveira e Afonso Yoshikiro Matsumoto, pela colaboração incansável na realização deste trabalho.

Aos meus colegas da Unidade Clínica de Miocardiopatias pela colaboração constante e fraterna durante todos os dias de minha vida Institucional: Barbara Maria lanni, Felix José Alvarez Ramires, Fábio Fernandes, Luciano Nastari e Vera Maria Cury Salemi.

À Lucia Maria de Oliveira, auxiliar direta na realização de toda minha produção científica e Roseli Chaves pelo apoio logístico.

Aos antigos e novos colaboradores do Grupo de Estudos da Cardiomiopatia Hipertrófica: Paula de Cássia Buck, Adriana Paula Tirone, Aloir Queiroz de Araujo, Afonso Akio Shiozaki, Milena Frota Macatrão Costa, Alexandre Pereira, José Eduardo Krieger, Júlia Daher Carneiro Marsiglia, Ligia M. Antunes Corrêa, Rodrigo Pedrosa e Fabricio Sanchez Bergamin. 
Esta tese está de acordo com:

Referências: adaptado de International Committee of Medical Journals Editors (Vancouver)

Universidade de São Paulo. Faculdade de Medicina. Serviço de Biblioteca e Documentação. Guia de apresentação de dissertações, teses e monografias. Elaborado por Anneliese Carneiro da Cunha, Maria Júlia de A. L. Freddi, Maria F. Crestana, Marinalva de Souza Aragão, Suely Campos Cardoso, Valéria Vilhena. São Paulo: Serviço de Biblioteca e Documentação; 2004.

Abreviaturas dos títulos dos periódicos de acordo com List of Journals Indexed in Index Medicus. 


\section{ÍNDICE}

ABREVIATURAS

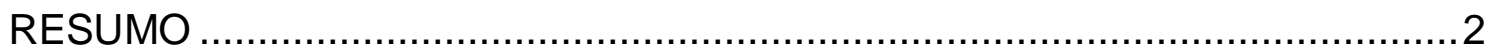

SUMMARY

INTRODUÇÃO

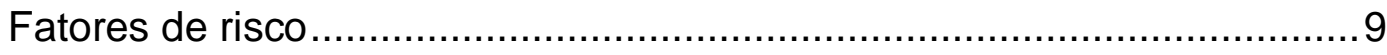

Prevenção de morte súbita na cardiomiopatia hipertrófica .......................12

Diretrizes de indicação de cardiodesfibrilador implantável .......................13

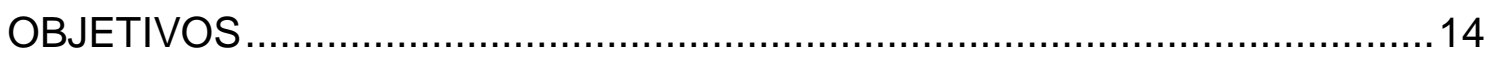

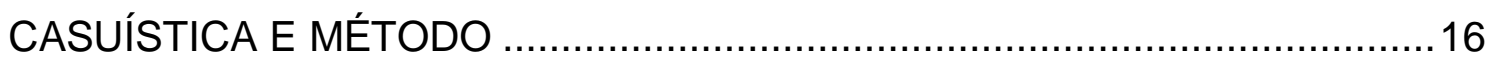

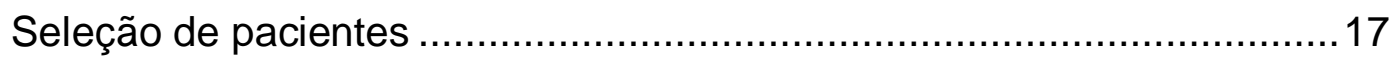

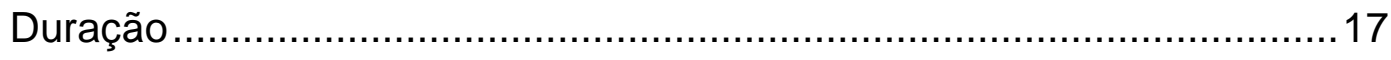

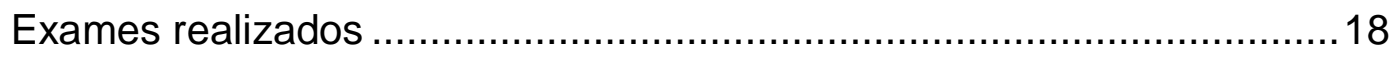

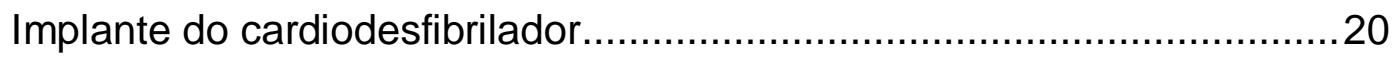

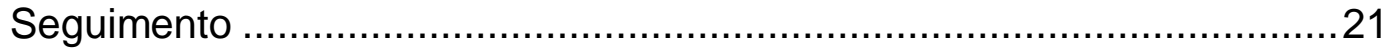

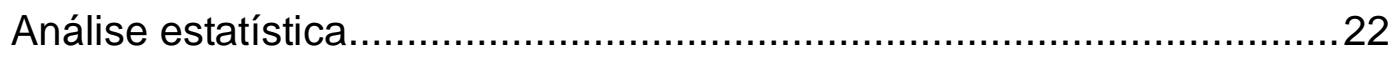

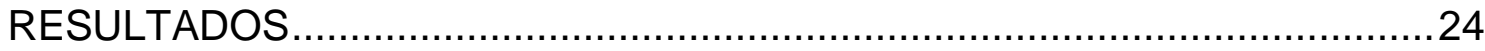

Caracterização clínica, eletrocardiográfica e ecocardiográfica..................25

Fatores de risco

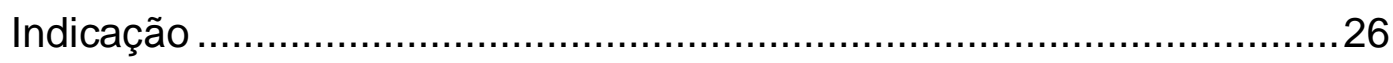

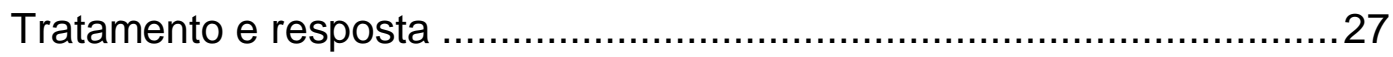

Choques apropriados e troca de cardiodesfibrilador implantável .............32

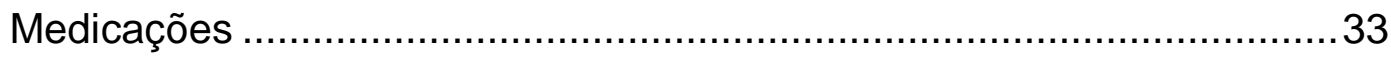

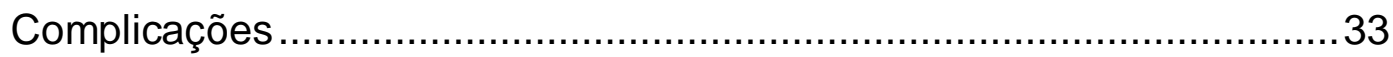

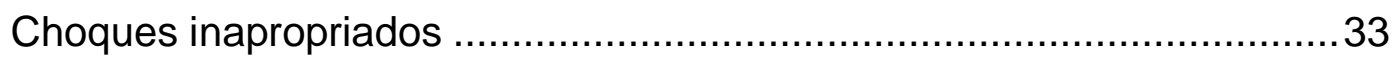

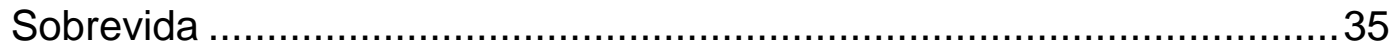

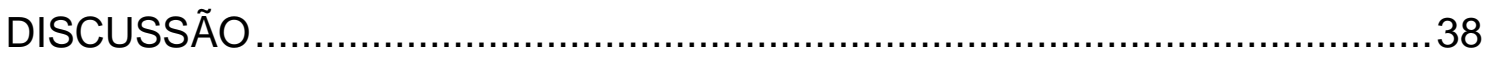

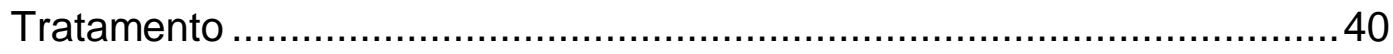

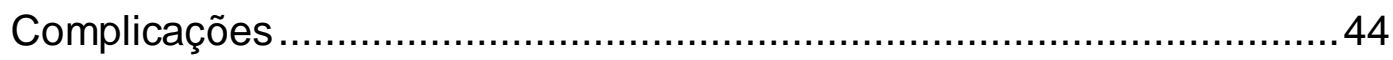

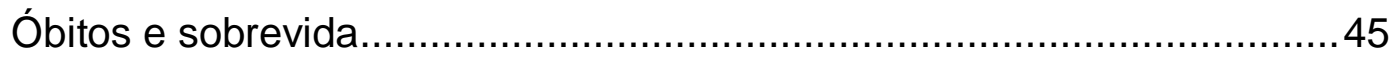

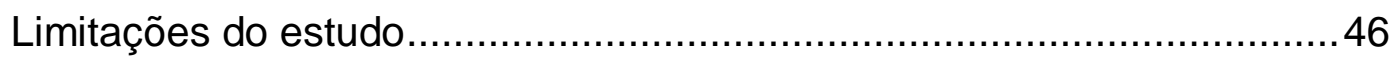

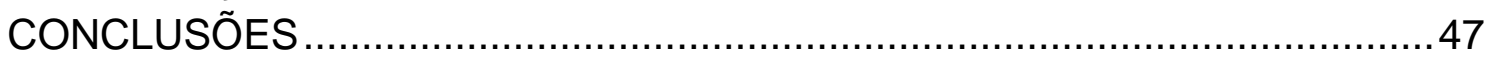

ANEXO

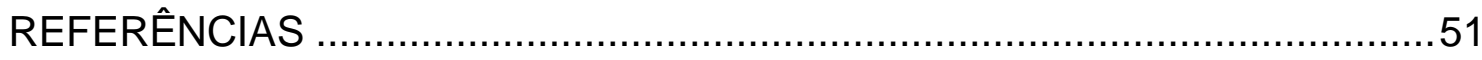




\section{ABREVIATURAS}

CDI - cardioversor-desfibrilador implantável

$\mathrm{CH}$ - cardiomiopatia hipertrófica

FV - fibrilação ventricular

$\mathrm{HR}$ - hazard ratio

IC - índice de confiança

MP - marcapasso cardíaco

MSC - morte súbita cardíaca

CF/NYHA - Classe funcional da New York Heart Association

OR - odds ratio

SIV - septo interventricular

TPSV - taquicardia paroxística supraventricular

TVNS - taquicardia ventricular não sustentada

TVS - taquicardia ventricular sustentada

VE - ventrículo esquerdo 


\section{RESUMO}

Arteaga-Fernández E. Cardiodesfibrilador implantável na prevenção de morte súbita na cardiomiopatia hipertrófica [tese]. São Paulo: Faculdade de Medicina, Universidade de São Paulo; 2011. 58p.

Com o objetivo de avaliar o efeito do cardiodesfibrilador implantável na sobrevida e as complicações por ele determinadas em pacientes com cardiomiopatia hipertrófica estudamos, de forma prospectiva, 61 (8\%) pacientes de uma coorte de 727 com diagnóstico de cardiomiopatia hipertrófica que receberam cardiodesfibrilador implantável para prevenção primária e secundária, matriculados no ambulatório da Unidade Clínica de Miocardiopatias do Instituto do Coração do Hospital das Clínicas da Faculdade de Medicina da Universidade de São Paulo, entre 2000 e 2010. A idade variou de 12 a 68 anos, com média de 37士15; 26 (43\%) pacientes eram do sexo masculino e $35(57 \%)$ do feminino. Os pacientes foram submetidos à eletrocardiografia, eletrocardiografia dinâmica de 24 horas, ecocardiografia bidimensional com Doppler colorido; seguimento a cada 6 meses e sempre que houve choques retornava à consulta para interrogação do aparelho. Cardiodesfibrilador implantável foi indicado como prevenção secundária em 15 (25\%) pacientes que tiveram parada cardíaca por taquicardia ventricular sustentada ou fibrilação ventricular documentada e prevenção primária em 46 (75\%) que tinham um ou mais dos seguintes fatores de risco: história de morte súbita em um ou mais parentes de $1^{\circ}$ grau com menos de 50 anos, síncope inexplicada, taquicardia ventricular não sustentada e hipertrofia miocárdica com espessamento do septo interventricular $>30 \mathrm{~mm}$. A análise estatística foi realizada por meio de cálculo de tendência central, dispersão e posição; teste de qui-quadrado de Pearson ou exato de Fisher para avaliar associações entre medidas qualitativas e teste de Mann-Withney para comparação entre medidas quantitativas. Óbitos, choques apropriados e inapropriado foram avaliados ao longo do tempo pela curva de sobrevida de Kaplan-Meier e comparadas pelo log-rank. Valores de $p<0,05$ foram considerados significantes. $O$ tempo médio de seguimento foi de $5 \pm 3$ anos (1 mês a 10 anos). Choques apropriados ocorreram em $17 / 61$ (28\%) pacientes, (média de 2 por paciente) e o tempo para o primeiro choque foi de 16 meses. Se considerarmos todo o grupo de 
pacientes, os que apresentam mais de 2 fatores de risco tiveram maior número de choques apropriados ( $p=0,04$ Fisher). Pacientes que tiveram choques apropriados apresentaram maior proporção de síncope ( $p=0,03$ Pearson). A proporção de choques apropriados no primeiro ano foi de $7 \%$, não diferindo entre os dois tipos de indicação ( $7 \%$ no grupo de prevenção secundária e $6 \%$ no de prevenção primária). As complicações foram observadas em 29/61 $(48 \%)$, sendo $25(41 \%)$ relacionadas ao sistema do aparelho e $8(13 \%)$ ao procedimento do implante. Os choques inapropriados ocorreram em 22/61(36\%) pacientes e o primeiro ocorreu depois de 23 meses. Observamos associação significante no sexo masculino ( $p=0,05$ Pearson) com presença de taquicardia ventricular não sustentada ( $p=0,03$ Pearson), com septo interventricular $>30 \mathrm{~mm}$ ( $p=0,05$ Pearson), uso de amiodarona ( $p=0,001$ Pearson) e troca de cardiodesfibrilador implantável. Nestes últimos a chance de choque inapropriado foi quatro vezes maior (OR=4,3; IC=95\%), observado apenas no grupo de prevenção primária. Observamos $6(10 \%)$ óbitos, sendo 5 (8\%) relacionados à doença e destes, 3 (60\%) faleceram subitamente, 1 por insuficiência cardíaca e 1 por acidente vascular cerebral. Podemos concluir que o tratamento com cardiodesfibrilador implantável na prevenção de morte súbita de pacientes com cardiomiopatia hipertrófica é eficiente, entretanto, ainda limitado pela elevada ocorrência de complicações do sistema geradorcabo-eletrodo, que determina choques inapropriados. Em nossa opinião, deve ser indicado na prevenção secundária e somente em casos selecionados, na prevenção primária.

Descritores: Desfibriladores implantáveis, Cardiomiopatia hipertrófica, Morte súbita cardíaca, Sobrevida, Fatores de risco, Prevenção primária, Prevenção secundária 


\section{SUMMARY}

Arteaga-Fernández E. Implantable cardioverter-defibrillator in preventing sudden death in hypertrophic cardiomyopathy [thesis]. São Paulo: "Faculdade de Medicina, Universidade de São Paulo"; 2011. 58p.

To evaluate the effect of implantable cardioverter-defibrillator on survival and complications of hypertrophic cardiomyopathy we prospectively studied 61 (8\%) patients, who received implantable cardioverter-defibrillator for primary and secondary prevention, from a cohort of 727 diagnosed with hypertrophic cardiomyopathy, enrolled at the outpatient clinic of Cardiomyopathies Unit of Heart Institute University of São Paulo Medical School between 2000 and 2010. The age ranged from 12 to 68 years old, median age $37 \pm 15.26(43 \%)$ patients were male and $35(57 \%)$ were female. Patients were submitted to electrocardiography, dynamic electrocardiographic monitored 24 hours, EchoDopplercardiography, follow-up every six months and whenever there is a query returning shocks for the interrogation device. The indication for cardioverter-defibrillator implantation was secondary prevention in 15 (25\%) patients that had cardiac arrest due to sustained ventricular tachycardia or ventricular fibrillation documented, and primary prevention in $46(75 \%)$ that had one or more of the following risk factors: history of sudden death in one or more first degree relatives younger than 50 years, unexplained syncope, nonsustained ventricular tachycardia and myocardial hypertrophy with thickening of the interventricular septum $>30 \mathrm{~mm}$. Statistical analysis was performed by statistics of central tendency, dispersion and position, the chisquare test or Fisher's exact test to assess associations between qualitative measures and the Mann-Whitney test for comparison of quantitative measures. Events deaths, inappropriate shock and therapy shocks were evaluated over time by the survival curve by Kaplan-Meier method and compared by Log-Rank. $P$ values $<0.05$ were considered significant. The mean follow-up was $5 \pm 3$ years ( 1 month to 10 years). The therapies shocks occurred in 17/61 (28\%) patients, with an average of 2 therapies shocks per patient and the time to first therapy was 16 months. Regarding the entire group of patients, those with more than two risk factors, had a greater rate of therapies shocks ( $p=0.04$ Fisher). Moreover, those who had therapy shocks, had higher rates of syncope $(p=0.03$ 
Pearson). The rates of therapies shocks in the first year were $7 \%$, no difference between the two types of statement $(7 \%$ in the secondary prevention group and $6 \%$ in primary prevention). Complications were observed in 29/61 (48\%) and 25 $(41 \%)$ was related to the implantable cardioverter-defibrillator system and 8 $(13 \%)$ to the surgical procedure. The inappropriate shocks occurred in $22 / 61$ (36\%), and the first shock occurred after 23 months. We observed a significant association between inappropriate shocks and male gender ( $p=0.05$ Pearson), presence of nonsustained ventricular tachycardia $(p=0.03$ Pearson), myocardial hypertrophy with interventricular septum $>30 \mathrm{~mm}$. $(p=0.05$ Pearson), use of amiodarone ( $p=0.001$ Pearson) and exchange of implantable cardioverter-defibrillator. The chance of these inappropriate shocks were four times higher among patients who exchanged implantable cardioverterdefibrillators, $(O R=4.3 \mathrm{Cl}=95 \%)$ but it happens only on the group of primary prevention. We observed that $6(10 \%)$ patients died: $5(8 \%)$ related to the disease due to sudden death (3-60\%), heart failure (1) and stroke (1). We conclude that treatment with implantable cardioverter-defibrillator on preventing sudden death in patients with hypertrophic cardiomyopathy is efficient. But it is still limited by the high incidence of complications of the system device, which may causes the inappropriate shocks. In our opinion it must be indicated in secondary prevention and only in selected cases for primary prevention.

Descriptors: Implantable defibrilators, Hypertrophic cardiomyopathy, Sudden cardiac death, Survivorship, Risk factors, Primary prevention and secondary prevention 
INTRODUÇÃO 
A cardiomiopatia hipertrófica $(\mathrm{CH})$ é doença primária do músculo cardíaco, cuja característica é hipertrofia sem dilatação ventricular na ausência de doença cardíaca, sistêmica ou metabólica que possa associar-se a hipertrofia. A cavidade do ventrículo esquerdo (VE) apresenta-se normal ou reduzida, havendo disfunção diastólica, enquanto a função sistólica em repouso é normal ou observa-se estado hiperdinâmico. Entretanto, a literatura mostra que em fase avançada da doença, até $10 \%$ dos pacientes evoluem com dilatação do VE e função sistólica diminuída ${ }^{1-5}$.

É doença genética autossômica dominante em $60 \%$ a $82 \%$ dos pacientes, com mais de 900 mutações em 13 genes codificadores de proteínas do sarcômero, além de recentes mutações descobertas em genes do disco Z e reguladores de cálcio que podem causar a doença 6,7 . Sua prevalência é estimada em $0,2 \%$ da população geral e em $0,5 \%$ entre os portadores de cardiopatia 8.

As alterações observadas em necrópsias incluem: hipertrofia do VE com espessamento maior do septo interventricular (SIV) em relação à parede livre; cavidade ventricular diminuída ou normal; átrio esquerdo dilatado e valva mitral com alterações em $66 \%$ dos casos, com ou sem espessamento secundário; placa de fibrose no SIV na via de saída do VE, no local do choque da cúspide anterior da valva mitral; artérias coronárias intramurais com espessamento da camada média e diminuição da luz em $80 \%$ dos casos; alteração da arquitetura miocárdica por hipertrofia e desarranjo das fibras, assim como das miofibrilas e da matriz extracelular, com aumento do colágeno e destruição das fibras elásticas ${ }^{3,4,} 9$.

Essas alterações anatômicas determinam um substrato que favorece o aparecimento de arritmias ventriculares. Assim, a hipertrofia causa dispersão 
da repolarização e alteração no período refratário aumentando a vulnerabilidade do miocárdio e início de arritmias ${ }^{9}$. As alterações das artérias coronárias são, possivelmente, responsáveis pela isquemia e fibrose que, associadas a áreas de desarranjo celular, geram áreas de bloqueio de condução que predispõem às arritmias por reentrada ${ }^{10}$. Anormalidades do fluxo de íons, principalmente do cálcio durante a repolarização, pode desencadear início de arritmias ${ }^{11}$. Esse complexo substrato arritmogênico pode ser modulado, ainda, por distúrbios de resposta autonômica, isquemia miocárdica e obstrução da via de saída do VE 5 .

Desde a descrição da $\mathrm{CH}$, inúmeros pesquisadores têm procurado estratificar o risco de morte súbita, que permanece ainda indefinido, apesar do avanço no conhecimento da doença e dos exames complementares disponíveis. Sabemos que a morte súbita cardíaca (MSC) ocorre entre 12 e 35 anos, sendo rara na primeira década de vida e após os 65 anos. Pode ser o primeiro sintoma e ocorre principalmente no período da manhã, durante exercício moderado ou durante o sono; exercício vigoroso pode ser fator desencadeante e está relacionado a taquiarritmias ventriculares. Sua incidência é $<1 \%$ /ano em pacientes sem fatores de risco e de $1,4 \%$ a $5,0 \%$ /ano naqueles com um ou mais fatores de risco $5,12,13$.

Os objetivos do tratamento da $\mathrm{CH}$ são o alívio dos sintomas e a prevenção de MSC. Nesse sentido, as opções farmacológicas disponíveis são os betabloqueadores, bloqueadores dos canais de cálcio, amiodarona 4, 5, 14 e, mais recentemente, drogas bloqueadoras da angiotensina II 15, que proporcionam controle dos sintomas e melhora da disfunção diastólica em aproximadamente $90 \%$ dos casos, mas falham ao não protegerem da MSC 16 . Para as formas obstrutivas, refratárias ao tratamento medicamentoso, as 
alternativas terapêuticas não-farmacológicas são a miectomia transvalvar aórtica e a terapia percutânea transcoronária de redução da hipertrofia miocárdica septal 5; esta última pode acarretar aumento de taquiarritmias ventriculares e morte súbita ${ }^{17}$.

\section{Fatores de risco}

A morte súbita cardíaca pode acontecer em qualquer momento da vida podendo, inclusive, ser a primeira manifestação da doença. Todavia, é mais freqüente no final da adolescência e em adultos jovens 5,12,13.

$\mathrm{Na}$ literatura são relatados e aceitos como fatores de alto risco para morte súbita cardíaca e têm elevada significância em pacientes com idade $<50$ anos $5,10,12,13,18,19$ :

1. pacientes que sobreviveram a parada cardíaca com fibrilação ventricular documentada ou que tiveram um episodio de taquicardia ventricular sustentada;

2. história familiar de morte súbita em um ou mais parentes de primeiro grau com menos de 50 anos;

3. síncope recente inexplicada (nos últimos 6 meses) - que ocorre em circunstâncias sem relação com evento de origem neurológica;

4. hipertrofia do miocárdio importante $>30 \mathrm{~mm}$ em qualquer segmento do miocárdio no ecocardiograma bidimensional;

5. presença de taquicardia ventricular não sustentada (TVNS) - três ou mais extrassístoles ventriculares consecutivas com freqüência $>120$ bpm no Holter; 
6. resposta anormal da pressão arterial ao exercício - aumento da PAS no ínicio até o pico $<25 \mathrm{mmHg}$ ou queda $>10 \mathrm{mmHg}$.

A história familiar de morte súbita, relacionada principalmente a mutações do gene da cadeia pesada da beta-miosina cardíaca e do gene da troponina $\mathrm{T}$, é bem conhecida e quiçá a variável de maior risco para o paciente individual. Vários trabalhos na literatura, embora com dados não muito robustos, têm demonstrado que esta variável aumenta em duas vezes o risco para morte súbita $7,18,19$.

Um episódio de síncope inexplicada pode ser considerado equivalente a morte súbita abortada em paciente com $\mathrm{CH}$, entretanto há que se levar em conta outros mecanismos que podem levar à perda da consciência como a obstrução da via de saída do VE, arritmias e a vasodilatação periférica causada por reflexo vascular anormal; mesmo assim, muitas vezes é difícil identificar de forma retrospectiva a causa da síncope. Num extenso trabalho com 1511 pacientes com $\mathrm{CH}$ foi observada síncope inexplicada em 153 (14\%) e nos que apresentaram síncope num período de 6 meses antes da avaliação inicial o risco de morte resultou cinco vezes maior se comparado ao grupo que não apresentou síncope $(H R=5 ; I C=95 \%)$. O risco é maior se o paciente for jovem com menos de 18 anos. Outro dado interessante é que a síncope pode ter relação com a obstrução de via de saída de VE uma vez que os pacientes que a tinham apresentaram mais frequentemente síncope de esforço 20.

A importante hipertrofia do VE determina aumento do consumo de oxigênio, isquemia do miocárdio e aumenta o risco de arritmias ventriculares complexas. Trabalhos têm demonstrado que pacientes com espessura da parede ventricular $>30 \mathrm{~mm}$ têm freqüência maior de morte súbita quando 
comparados a controles ou pacientes com espessura $<20 \mathrm{~mm}$. O perfil clínico destes pacientes é que são jovens com menos de 31 anos, pouco sintomáticos e com risco importante de morte súbita ${ }^{21,22 . ~ A ~ a v a l i a c ̧ a ̃ o ~ d a ~ m a s s a ~ v e n t r i c u l a r ~}$ esquerda pela ressonância magnética mostrou que quanto maior a massa menor a sobrevida de pacientes com $\mathrm{CH}^{23}$.

Vários estudos mostraram que pacientes com um ou dois episódios de taquicardia ventricular não sustentada (TVNS) com frequência de 120 batimentos/minuto ou mais ao Holter têm risco relativo de morte súbita cardíaca de 2 a 2,5 vezes maior. Esses pacientes tinham menos de 30 anos, apresentavam 1 a 2 episódios de TVNS ao Holter e encontravam-se em classe funcional III/IV da New York Heart Association (CF/NYHA). Observou-se que acima dessa idade a prevalência de TVNS aumenta, mas o risco relativo de MSC diminui. A prática clínica mostra que um episódio curto de TVNS (três batimentos) não indica a colocação de CDI; entretanto, episódios repetitivos de TVNS prolongada (mais de 10 batimentos) podem ter risco maior 14, 24, 25.

A resposta anormal da pressão arterial ao exercício é observada em $1 / 3$ dos pacientes com $\mathrm{CH}$ e tem relação com MSC. Este teste não é realizado frequentemente em comparação aos outros e raramente é utilizado como fator único na indicação de CDI 5,18.

Nos últimos anos, estudos com ressonância magnética têm mostrado que pacientes com realce tardio (fibrose) apresentam maior frequência de TVNS ao Holter e maior mortalidade 26,27 . Observamos em estudo de biópsia endomiocárdica do ventrículo direito pela avaliação da fração de volume de colágeno pelo picriosirius que os pacientes que vieram a falecer num seguimento médio de 10 anos tinham maior volume de colágeno 28 . 
Outros fatores que podem associar-se aos já descritos e contribuir para aumentar o risco de morte súbita são: fase avançada da doença com disfunção diastólica em fase restritiva ou disfunção sistólica ${ }^{29}$; aneurisma apical do ventrículo esquerdo ${ }^{30}$; gradiente elevado na via de saída do ventrículo esquerdo (VE) ${ }^{31}$. Pacientes submetidos a tratamento de terapia transcoronária de redução da hipertrofia septal apresentam maior número de taquiarritmias ventriculares sustentadas e aumento de morte súbita após o mesmo ${ }^{32}$. Um fator de risco modificável é a prática de esportes competitivos em pacientes com $\mathrm{CH}$, mesmo que não tenham os fatores de risco convencionais.

\section{Prevenção da morte súbita na cardiomiopatia hipertrófica}

Em 1980 foi realizado o primeiro implante de cardiodesfibrilador implantável (CDI) por Mirowski et al. ${ }^{33}$ para prevenção de morte súbita cardíaca e em 2000 é demonstrada a eficácia em pacientes com $\mathrm{CH}$ e risco elevado de MSC 34 .

O CDI é efetivo no tratamento das taquiarritmias ventriculares, pois controla a fibrilação ventricular (FV) em $98 \%$ dos casos; com a função de marcapasso (MP) pode tratar de doença do nó sinusal e distúrbios de condução acentuados pelo uso concomitante de medicação antiarrítmica; pode, ainda, reduzir o gradiente da via de saída do VE e tratar a fibrilação atrial paroxística, que é observada em $25 \%$ dos pacientes com $\mathrm{CH}$. Tem demonstrado que salva vidas quando utilizado como prevenção secundária (pacientes recuperados de parada cardíaca ou com taquiarritmias ventriculares sustentadas) e há consenso universal da indicação nesta situação e profilaticamente em 
pacientes com alto risco de morte súbita - prevenção primária ${ }^{34-40}$. Também têm sido relatadas suas desvantagens, tais como, o alto custo, duração limitada da bateria, choques inapropriados em $25 \%$ dos pacientes, falha anual de 3\% e complicações do sistema de estimulação e do procedimento cirúrgico de implante 40,41 .

\section{Diretrizes de indicação de CDI}

As últimas diretrizes para implante de CDI das Sociedades Européia e Americana (2006) e Brasileira de Cardiologia (2007) ${ }^{42-44}$ preconizam o uso do CDI como prevenção secundária: Classe I - nível de evidência B, para pacientes com TVS/FV ou que foram recuperados de parada cardíaca. Nesta indicação, de cada 100 pacientes que implantaram CDI, 11 tiveram choques apropriados no $1^{\circ}$ ano ${ }^{18,38}$. Na prevenção primária: Classe lla - nível de evidência C, para os que apresentam um ou mais fatores preditores de MSC na $\mathrm{CH}$. Nesta indicação, de cada 100 pacientes que implantaram CDI, apenas 4 tiveram choque apropriados no $1^{\circ}$ ano ${ }^{18}$.

Hoje o maior desafio clínico é a identificação desse pequeno subgrupo de pacientes que pode ter complicações importantes, culminando com a evolução rápida da doença, ou mesmo com morte súbita.

Outro desafio é quanto à indicação na prevenção primária, diga-se de passagem, não uniforme entre os estudiosos da doença, já que o custo é elevado, bem como a alta taxa de complicações. Isto nos motivou a avaliar o efeito do CDI na sobrevida de nossos pacientes com $\mathrm{CH}$. 


\section{OBJETIVOS}


1. Avaliar o efeito do cardiodesfibrilador implantável na prevenção de morte súbita cardíaca em pacientes portadores de cardiomiopatia hipertrófica.

2. Avaliar a taxa de utilização do cardiodesfibrilador implantável quando da indicação na prevenção primária e secundária.

3. Avaliar a relação entre os preditores de morte súbita cardíaca e a indicação na prevenção primária e secundária.

4. Avaliar as relações entre choques apropriados do cardiodesfibrilador implantável com os preditores de morte súbita e as variáveis clínicas e demográficas.

5. Avaliar a incidência de complicações relacionadas ao tratamento. 
CASUÍSTICA E MÉTODO 
De uma coorte de 727 pacientes com diagnóstico de $\mathrm{CH}$ em seguimento na Unidade Clínica de Miocardiopatias do Instituto do Coração do Hospital das Clínicas da Faculdade de Medicina da Universidade de São Paulo, foram estudados, de forma prospectiva, $61(8 \%)$ que tiveram indicação pela presença de fatores de risco de morte súbita cardíaca e colocaram CDI, após decisão em conjunto com o Serviço de Estimulação Cardíaca Artificial (protocolo de pesquisa aprovado pela Comissão de Ética para Análise de Projetos de Pesquisa sob no. 0383/10, anexo).

\section{Seleção de pacientes}

Os pacientes tiveram diagnóstico clínico realizado no próprio hospital por meio de anamnese, exame físico e confirmado pela ecocardiografia que evidenciou hipertrofia ventricular com espessamento de parede $>15 \mathrm{~mm}$, na ausência de outra causa clínica. Para indicação do CDI foram considerados os seguintes fatores de risco: na prevenção secundária - parada cardíaca com fibrilação ventricular documentada ou que tiveram um episódio de taquicardia ventricular sustentada; na prevenção primária - história de morte súbita em um ou mais parentes de $1^{\circ}$ grau com menos de 50 anos, síncope inexplicada afastada a origem neurocardiogênica, TVNS no Holter e hipertrofia miocárdica com espessamento da parede do VE $>30 \mathrm{~mm}$ no estudo ecocardiográfico. Os pacientes poderiam apresentar um, dois, três ou até quatro fatores de risco.

\section{Duração}

O estudo iniciou-se em $1^{\circ}$ de março de 2001 , na ocasião do implante do primeiro CDI e foi encerrado em 31 de agosto de 2010. 


\section{Exames realizados}

Todos os pacientes foram submetidos às seguintes avaliações:

a. Anamnese e exame físico - consideraram-se os seguintes sintomas: presença ou não de dispnéia, palpitações, precordialgia e síncope. Pela intensidade dos sintomas os pacientes foram classificados funcionalmente de acordo com os critérios da CF/NYHA. Foram considerados os seguintes sinais: presença ou não de sopro da via de saída do VE elou de insuficiência mitral, pulso e pressão arterial sistólica e diastólica, medida na posição supina. Os pacientes foram considerados como tendo a forma familiar quando a doença estava presente em algum parente de $1^{\circ}$ grau com diagnóstico clínico e ecocardiográfico.

b. Avaliação eletrocardiográfica - foi realizado eletrocardiograma de repouso nas 12 derivações clássicas em todos os pacientes.

c. Eletrocardiografia dinâmica de 24 horas pelo sistema Holter: os pacientes foram submetidos a monitorização eletrocardiográfica ambulatorial de 24 horas, com uso de gravadores portáteis Marquette 8000 , com calibração fixada em $1 \mathrm{mV}$ : $10 \mathrm{~mm}$, registro de ondas com amplitude modulada (AM) e gravação contínua, para posterior análise em equipamento Holter GEMarquette, MARS-versão 4.0a. As variáveis analisadas foram: freqüência cardíaca média, número de extrassístoles supraventriculares por hora, presença ou não de taquicardia paroxística supraventricular (TPSV) sustentada e não sustentada, ocorrência de fibrilação atrial, número e tipo de extrassístoles ventriculares e presença ou não de TVNS (3 ou mais batimentos consecutivos com freqüência cardíaca $>120$ bpm). 
d. Estudo Doppler-ecocardiográfico - foi realizado estudo ecocardiográfico bidimensional, modo-M e Doppler (pulsátil, contínuo e mapeamento de fluxo em cores), com a utilização de aparelho Acuson, modelo Sequoia (Mountain View, California), obtendo-se os cortes para-esternais e apicais padrão. Os registros ecocardiográficos do modo-M e do Doppler foram orientados pelo exame bidimensional e seguiram as recomendações da Sociedade Americana de Ecocardiografia 46. Para o diagnóstico ecocardiográfico da $\mathrm{CH}$ utilizaram-se critérios já bem definidos na literatura 5. As medidas foram realizadas em três ciclos cardíacos consecutivos, sendo os valores calculados pela média simples dessas medidas:

- Medidas básicas do ecocardiograma modo-M

- diâmetro atrial esquerdo em mm

- espessura diastólica da parede posterior do VE em $\mathrm{mm}$

- espessura diastólica do septo interventricular em $\mathrm{mm}$

- diâmetros sistólico e diastólico final do VE em $\mathrm{mm}$.

- Valores calculados a partir das medidas básicas do modo-M

- fração de ejeção pela fórmula de Teicholz ${ }^{47}$ :

$$
\begin{aligned}
& V d=D 3 \times(7 / 2,4+D) \\
& V s=S 3 \times(7 / 2,4+S) \\
& F E=V d-V s / V d
\end{aligned}
$$

- Medidas básicas ao bidimensional

- espessura diastólica da parede com maior espessura em mm.

- Medidas básicas ao Doppler

- onda-E = pico de velocidade do fluxo protodiastólico transmitral

- onda-A = pico de velocidade do fluxo telediastólico transmitral

- relação $E / A$ = relação entre as velocidades da onda-E e onda-A 
- tempo de relaxamento isovolumétrico do VE

- tempo de desaceleração da onda-E.

Foram utilizados critérios de normalidade já estabelecidos para as medidas das dimensões ventriculares. Em relação à função sistólica global do ventrículo esquerdo foram considerados como normais valores para a fração de encurtamento acima de $30 \%$ e para a fração de ejeção acima de 0,65 .

\section{Implante do cardiodesfibrilador (CDI)}

As indicações corresponderam aos critérios de inclusão do estudo, que estão contidos nas Diretrizes da Sociedade Brasileira de Arritmias Cardíacas (SOBRAC) ${ }^{44}$.

Os sistemas de estimulação utilizados foram de dupla-câmara em 44 pacientes e unicameral em 17, obedecendo-se à técnica convencional de implante de MP endocárdico com utilização de radioscopia. Após punção da veia subclávia esquerda foram implantados dois cabos-eletrodos bipolares endocárdicos, um de fixação ativa tipo screw-in posicionado na região do apêndice atrial direito, e um de fixação passiva com aletas e pólo para cardioversão ou desfibrilação na região da ponta do ventrículo direito. Nos CDI unicamerais apenas foi implantado o cabo ventricular.

Em seguida, foram realizadas as medidas perioperatórias das funções anti-bradicardia: limiares de estimulação em átrio e ventrículo, amplitudes da onda P e dos complexos QRS e impedâncias. Para as funções anti-taquicardia, realizava-se o choque de 1 Joule no cabo-eletrodo de cardioversão ou desfibrilação, para verificação da impedância do sistema e induzia-se FV com 
estimulação ventricular programada para determinação do limiar de desfibrilação ventricular.

O gerador do CDI foi implantado numa loja subcutânea localizada na região infraclavicular esquerda após a conexão dos cabos-eletrodos com os canais respectivos (atrial e ventricular). Foram utilizados geradores dos quatro fabricantes disponíveis no Brasil: Biotronik, Guidant, Medtronic e Saint Jude.

A programação do CDI foi protocolar: freqüência de estimulação 40 bpm para a zona de bradicardia (função de MP), com três zonas de detecção de taquicardia:

- Zona 1 - frequência entre 140 e 160 bpm

- Zona 2 - frequência entre 160 e 180 bpm

- Zona 3 - frequência superior a 180 bpm.

Para as duas primeiras, as funções de terapia foram desativadas e o CDI funcionava como monitor de registros para todo evento que ocorresse entre estas faixas de frequência, que foi chamada de Zona de Holter.

$\mathrm{Na}$ Zona 3 foi ativada a função de terapia com choque (cardioversão e/ou desfibrilação). A energia dos choques foi programada de acordo com o limiar de desfibrilação obtido na cirurgia.

\section{Seguimento}

Constou de avaliação clínica e do CDI a cada 6 meses, ou sempre que o paciente manifestasse síncope ou referisse choque do CDI. Os parâmetros avaliados foram: limiar de estimulação e de sensibilidade, impedância dos cabos-eletrodos $\mathrm{e}$ as condições da bateria. Os eventos registrados e 
armazenados pelo CDI foram resgatados na forma de eletrograma intracardíaco, incluindo o registro do canal de marcas. Considerou-se choque apropriado a arritmia potencialmente letal, taquicardia ventricular sustentada (TVS) ou fibrilação venticular (FV) detectadas pelo CDI, que foram revertidas por choque apropriado de cardioversão ou de desfibrilação e choque inapropriado aquele que foi desencadeado por taquicardia sinusal, fibrilação atrial ou falha por funcionamento defeituoso do dispositivo.

Todos os pacientes foram avaliados até 31 de agosto de 2010; nos pacientes que foram a óbito, obtivemos informações sobre o motivo e a forma do mesmo, do médico que atendeu em pronto socorro e dos familiares próximos ou cuidadores. Foi definido o tipo de óbito - se por causa cardíaca (morte súbita, insuficiência ou acidente vascular cerebral por fenômeno tromboembólico), não cardíaca, ou óbito de causa desconhecida. Morte súbita foi definida quando ocorreu até uma hora após o início dos sintomas.

\section{Análise estatística}

A amostra estudada e constituída por 61 pacientes teve suas características resumidas através de estatísticas descritivas de frequências (absolutas e relativas), das medidas qualitativas e estatísticas de tendência central (média e mediana), dispersão (desvio padrão - DP, mínimo e máximo) e posição (percentis 25 e 75) das medidas quantitativas.

Testes de qui-quadrado de Pearson ou exato de Fisher foram aplicados para avaliação da associação entre medidas qualitativas. O teste não paramétrico de Mann-Whitney foi aplicado para comparação de medidas 
quantitativas. Foram considerados estatisticamente significantes os resultados cujos níveis descritivos (valores de $p$ ) foram inferiores a 0,05 .

Os eventos: óbito, choque apropriado e inapropriado foram avaliados ao longo do tempo pela curva de sobrevida de Kaplan-Meier e curvas de sobrevida comparadas entre grupos pelo teste de log-rank.

As variáveis estudadas foram:

- Clínicas: idade, sexo, síncope, classe funcional, morte súbita familiar, indicação do CDI, sobrevida e óbito

- Medicações: betabloqueadores, verapamil e amiodarona

- Eletrocardiográficas: fibrilação atrial, taquicardia ventricular não sustentada, taquicardia ventricular sustentada e fibrilação ventricular

- Ecocardiográficas: gradiente de via de saída do VE, hipertrofia miocárdica $>30 \mathrm{~mm}$

- Outras: choques apropriados, choques inapropriados, complicações do sistema (dispositivo e cabo-eletrodo) e do procedimento de colocação e troca de CDI. 
RESULTADOS 


\section{Caracterização clínica, eletro e ecocardiográfica (tabela 1)}

O tempo de seguimento médio foi de $5 \pm 3$ anos [(30 dias a 10 anos) (percentil $25=3$ anos e percentil $75=8$ anos)].

Dos 61 pacientes estudados, 35 (57\%) eram do sexo feminino e 26 (43\%) do masculino, com idades entre 12 e 68 anos (média $37 \pm 15$ ), por ocasião da colocação do CDI.

A maioria dos pacientes 51 (84\%) eram pouco sintomáticos, estavam em CF I - $34(56 \%)$, II - 17 (28\%) e $10(16 \%)$ estavam em CF III. Todos estavam recebendo medicações: propranolol em doses diárias que variaram de 160 a $320 \mathrm{mg}$, atenolo/100 a $200 \mathrm{mg}$, verapamil 240 a $320 \mathrm{mg}$ e amiodarona 200 a 400 mg.

Ao eletrocardiograma observou-se fibrilação atrial em 13 (21\%) pacientes e ao Holter de 24 horas foi observada TVNS em 25 (41\%) pacientes.

Ao estudo ecocardiográfico observamos os seguintes valores: espessura do septo interventricular $24 \pm 7 \mathrm{~mm}$ (15-39), diâmetro do átrio esquerdo $45 \pm 8 \mathrm{~mm}$ (28-67), do ventrículo esquerdo em diástole $44 \pm 8 \mathrm{~mm}$ (3069), fração de ejeção $0,70 \pm 0,10(0,28-0,87)$ e gradiente da via de saída do VE $>30 \mathrm{mmHg}$ em 20 pacientes (33\%).

\section{Fatores de risco}

Dos 61 pacientes estudados, $43(70 \%)$ referiram história de morte súbita familiar e 41 (67\%) síncope de origem indeterminada; em 25 (41\%) foi observada TVNS, em 16 (26\%) hipertrofia miocárdica >30 mm e em 15 (25\%) TVS ou FV. Uma paciente da nossa Instituição que não apresentava fatores de risco teve indicação e colocação do CDI em outro hospital. Observamos 14 
pacientes (23\%) com 1 fator de risco, 26 (43\%) com 2 fatores de risco, $18(29 \%)$ com 3 fatores de risco e $2(3 \%)$ com 4 fatores de risco.

\section{Indicação}

CDI foi indicado como prevenção primária em 46 pacientes (75\%) e secundária em 15 pacientes (25\%).

Tabela 1 - Características clínicas, demográficas e dos exames complementares

\begin{tabular}{|c|c|c|c|c|}
\hline \multirow{2}{*}{\multicolumn{3}{|c|}{ Idade média (DP) }} & $n=61$ & $(\%)$ \\
\hline & & & $37 \pm 15(12-68)$ anos & \\
\hline \multirow[t]{2}{*}{ Sexo } & masculino & & 26 & 43 \\
\hline & feminino & & 35 & 57 \\
\hline \multirow{3}{*}{\multicolumn{2}{|c|}{ Classe funcional (NYHA) }} & 1 & 34 & 56 \\
\hline & & II & 17 & 28 \\
\hline & & III & 10 & 16 \\
\hline \multirow{4}{*}{\multicolumn{3}{|c|}{$\begin{array}{l}\text { ECO - septo interventricular }(\mathrm{mm}) \\
\text { diâmetro de atrio esquerdo }(\mathrm{mm}) \\
\text { diâmetro do ventrículo esquerdo }(\mathrm{mm}) \\
\text { fração de ejeção do ventriculo esquerdo }\end{array}$}} & $24 \pm 7(15-39)$ & \\
\hline & & & $45 \pm 8(28-67)$ & \\
\hline & & & $44 \pm 8(30-69)$ & \\
\hline & & & $70 \pm 10(28-87)$ & \\
\hline \multicolumn{3}{|c|}{ Formas obstrutivas } & 20 & 33 \\
\hline \multicolumn{3}{|c|}{ Fibrilação atrial } & 13 & 21 \\
\hline \multicolumn{3}{|c|}{ Indicação de CDI - prevenção primária } & 46 & 75 \\
\hline \multicolumn{3}{|c|}{ Historia familiar de morte súbita } & 43 & 70 \\
\hline \multicolumn{3}{|c|}{ Síncope inexplicada } & 41 & 67 \\
\hline \multicolumn{3}{|c|}{ Taquicardia ventricular não sustentada } & 25 & 41 \\
\hline \multicolumn{3}{|c|}{ Hipertrofia miocárdica $>30 \mathrm{~mm}$} & 16 & 26 \\
\hline \multicolumn{3}{|c|}{ Indicação de CDI - prevenção secundária } & 15 & 25 \\
\hline \multicolumn{3}{|c|}{ Taquicardia ventricular sustentada ou FV } & 15 & 25 \\
\hline \multirow{4}{*}{\multicolumn{2}{|c|}{ Número de fatores de risco }} & 1 & 14 & 23 \\
\hline & & 2 & 26 & 43 \\
\hline & & 3 & 18 & 29 \\
\hline & & 4 & 2 & 3 \\
\hline \multirow{3}{*}{\multicolumn{3}{|c|}{$\begin{aligned} & \text { Medicação - } \text { betabloqueador } \\
& \text { amiodarona } \\
& \text { Verapamil } \\
&\end{aligned}$}} & 52 & 85 \\
\hline & & & 32 & 52 \\
\hline & & & 22 & 36 \\
\hline
\end{tabular}




\section{Tratamento e resposta}

Os choques apropriados foram observados em 17/61 pacientes (28\%) e o primeiro ocorreu em média $16(1$ - 42) meses após o implante do CDI (percentil $25=3$ meses e percentil $75=28$ meses). A média de choques apropriados por paciente foi de $2(1-8)$; 10 pacientes $(59 \%)$ receberam 1 choque apropriado; 3 (18\%) 2; $1(6 \%) 3 ; 1(6 \%) 5$ e $2(12 \%) 8$ choques apropriados.

A proporção de choque apropriados no primeiro ano foi de $7 \%$, não diferindo significativamente entre os dois tipos de indicação. Numa estimativa no primeiro ano, em 100 implantes de CDI ocorreram 6 choques apropriados no grupo de prevenção primária e 7 no de prevenção secundária (figura 1 e tabela 2).

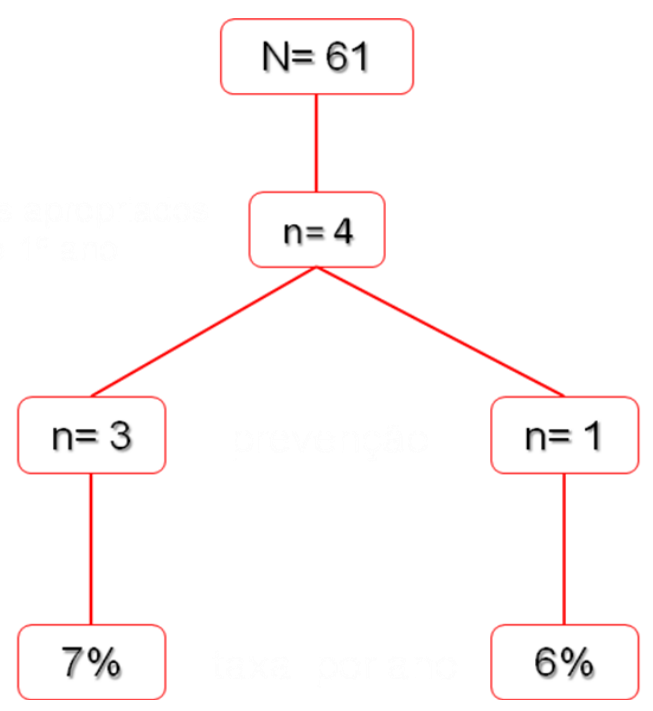

Figura 1 - Taxa de choques apropriados por indicação no $1^{\circ}$ ano 
Tabela 2 - Taxa de choques apropriados por indicação no $1^{\circ}$ ano $(p=0,68$ Fisher)

\begin{tabular}{c|c|c|c|c}
\hline \multicolumn{2}{c|}{} & Primária & Secundária & Total \\
\hline \multirow{4}{*}{ Choques apropriados } & Não & 43 & 14 & 57 \\
\cline { 2 - 5 } & Sim & 3 & 1 & 4 \\
\cline { 2 - 5 } & Indicação & $6 \%$ & $7 \%$ & $7 \%$ \\
\hline \multicolumn{2}{c}{ Total } & 46 & 15 & 61 \\
\hline
\end{tabular}

Ao analisar o número de choques apropriados por faixa etária, observamos maior proporção nos pacientes com 25 anos ou menos e naqueles com idade superior a 40 anos; entretanto, não houve evidência de diferença estatisticamente significante ( $p=0,92$ Fisher) das proporções entre as faixas etárias (figura 2).

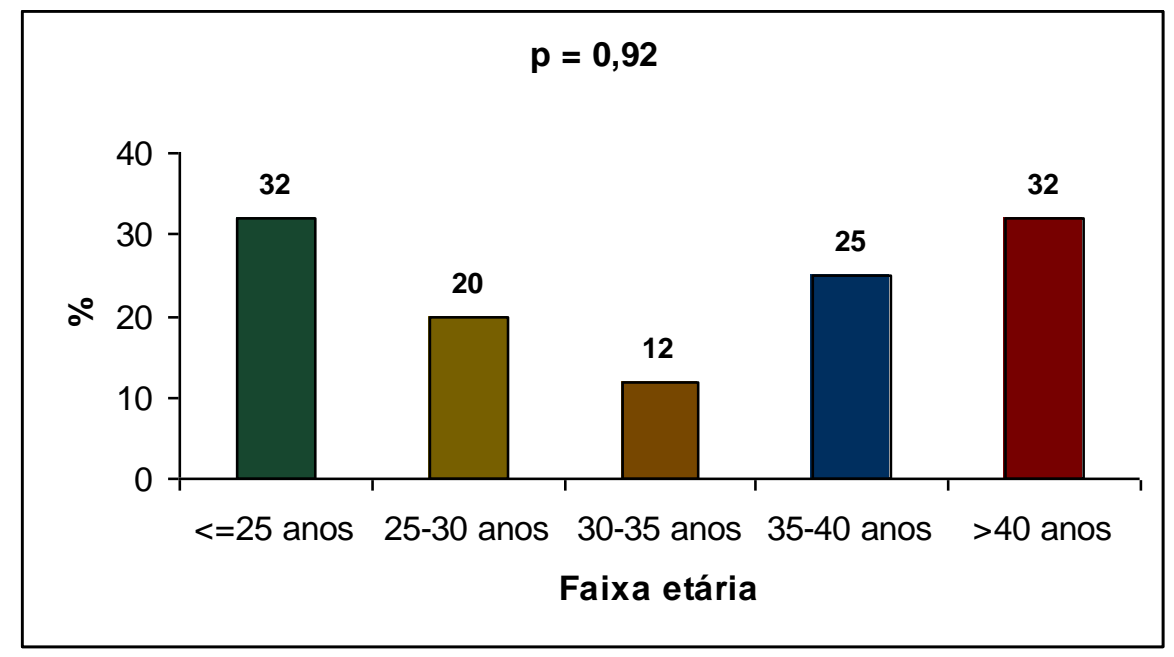

Figura 2 - Número de choques apropriados por faixa etária

A análise dos choques apropriados e o número de fatores de risco não mostrou diferença estatisticamente significante $(p=0,14$ Fisher) entre os grupos; entretanto, quando separamos em dois grupos: com menos ou mais de 2 fatores de risco, observamos que há diferença estatisticamente significante ( $p=0,05$ Fisher) na proporção de choques apropriados entre os que tiveram 
menos de 2 fatores de risco $(1 / 15=7 \%)$ e os que tiveram 2 ou mais $(16 / 46=$ $35 \%$ ) (figuras 3 e 4 ).

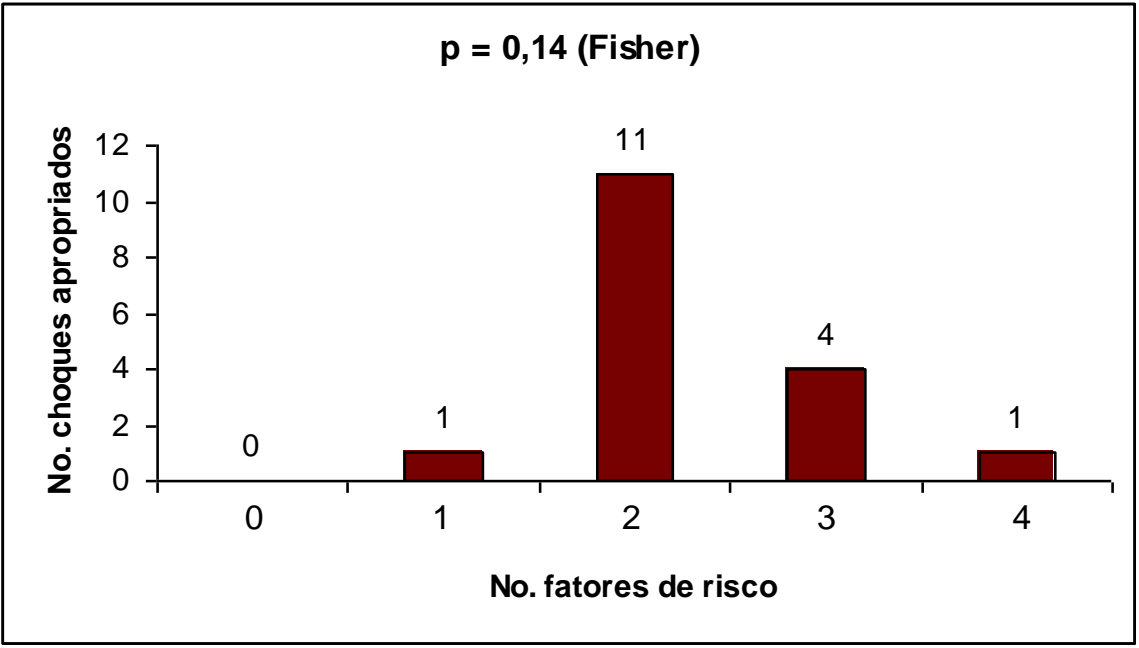

Figura 3 - Choques apropriados e número de fatores de risco

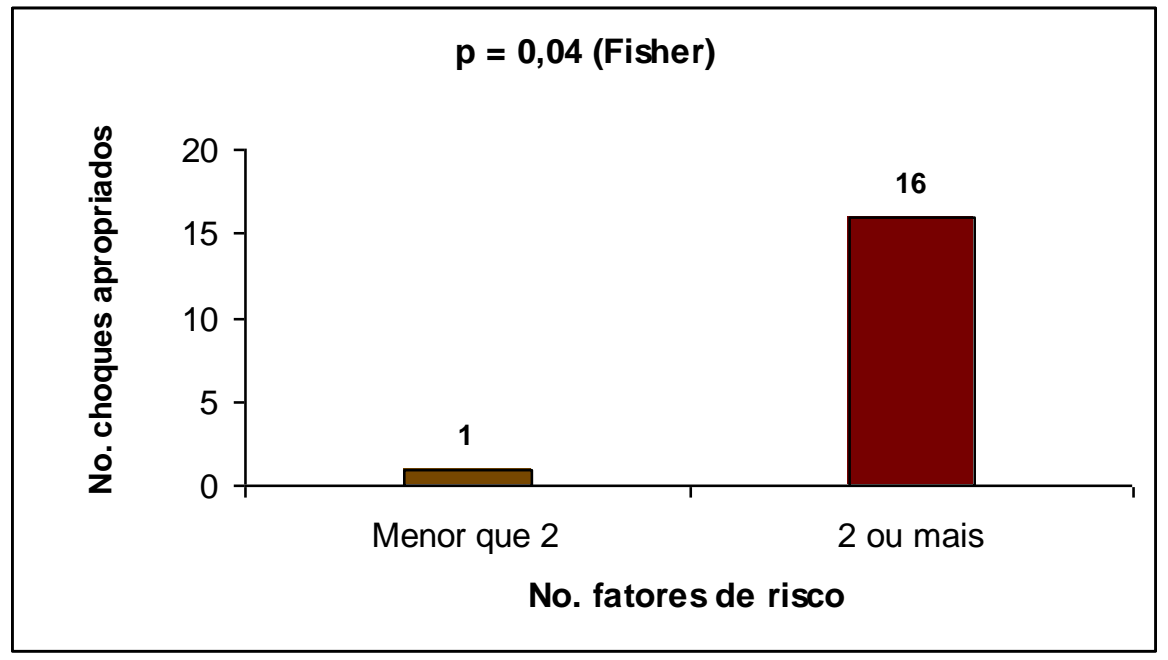

Figura 4-Choques apropriados e número de fatores de risco agrupados (com menos de 2 e mais de 2 )

Quando analisamos o choque apropriado e o número de fatores de risco por indicação não observamos diferença estatisticamente significante entre eles ( $p=0,55$ e $p=0,22$ Fisher), mesmo quando agrupados com menos de 2 ou mais de 2 fatores de risco na prevenção primária ( $p=0,25$ Fisher) ou secundária ( $p=0,10$ Fisher) (figuras 5 e 6). 


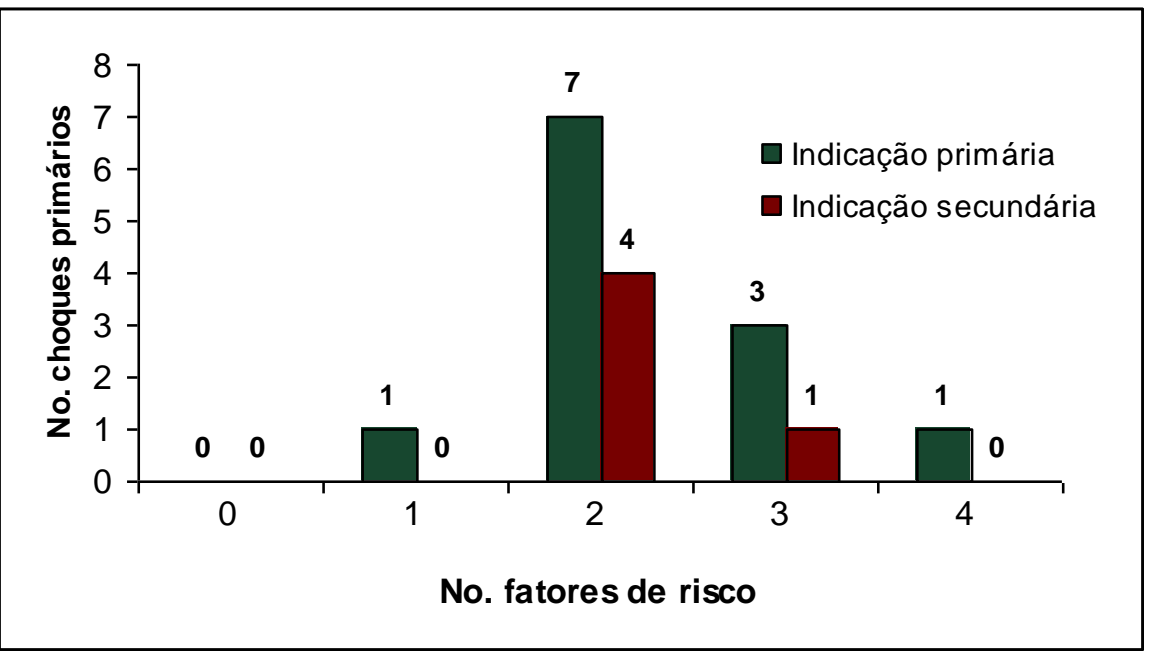

Figura 5 - Choque apropriado, número de fatores de risco e prevenção primária ( $p=0,55$ Fisher) e secundária ( $p=0,22$ Fisher).

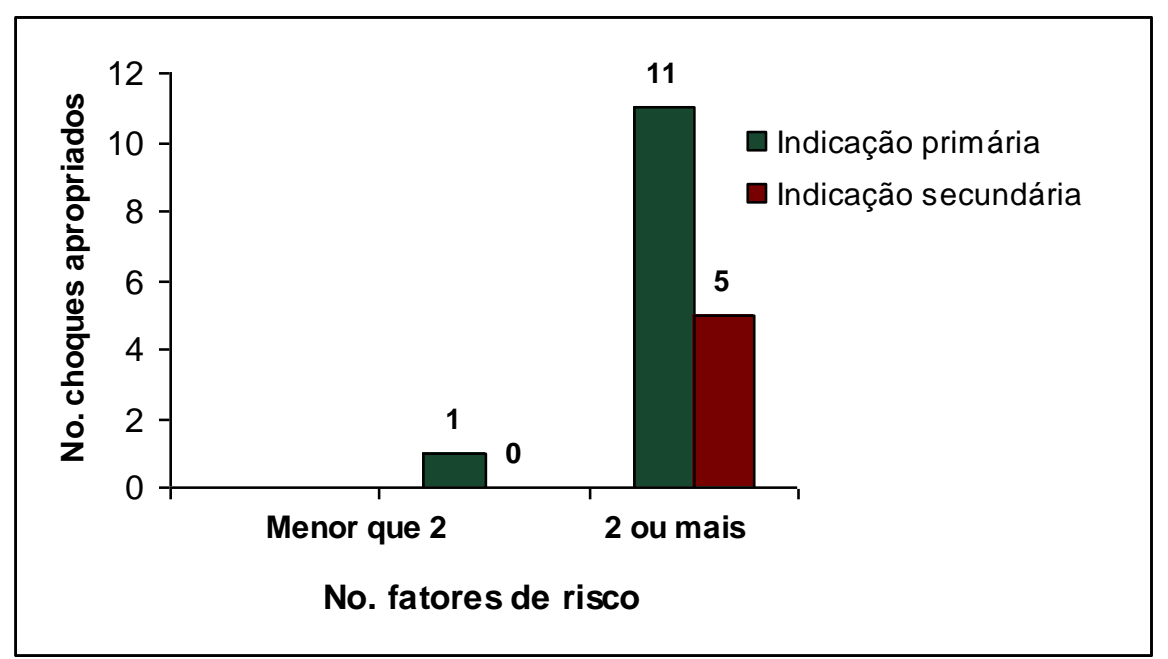

Figura 6 - Choques apropriados, número de fatores de risco e prevenção primária ( $p=0,25$ Fisher) e secundária ( $p=0,10$ Fisher)

Ao analisar a relação entre o choque apropriado e fatores de risco observa-se presença estatisticamente significante de choques apropriados (37\%) nos pacientes que tiveram síncope, em relação aos que não tiveram este fator de risco ( $p=0,03$ Pearson). Para os demais fatores não houve diferença estatisticamente significante nas proporções de choques apropriados entre o grupo com o fator de risco presente e o grupo com ausência do fator (figura 7). 


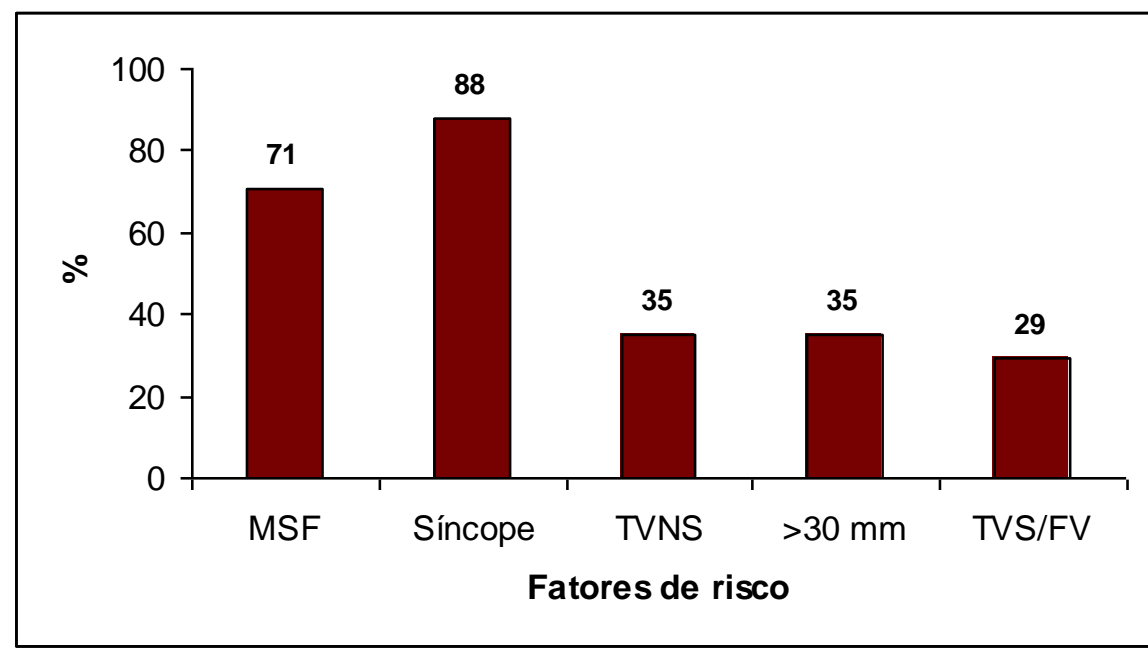

Figura 7 - Associação entre choque apropriado e fatores de risco

Quando analisamos a associação entre o número de choques apropriados e os fatores de risco observamos associação estatisticamente significante $(p=0,01)$ entre a quantidade de choques apropriados e taquiarritmias ventriculares, devido ao excesso dessas nos que tiveram 8 choques apropriados (100\%) e à falta desse fator nos que tiveram 1 choque apropriado (10\%) (tabela 3).

Tabela 3 - Distribuição de freqüências dos fatores de risco nas categorias da quantidade de choques apropriados

\begin{tabular}{c|c|c|c|c|c}
\hline $\begin{array}{c}\mathrm{N}^{\circ} \text { de } \\
\text { choques } \\
\text { apropriados }\end{array}$ & MSF & Síncope & TVNS & SIV >30mm & TVS ou FV \\
\hline 1 & $7(70 \%)$ & $9(90 \%)$ & $4(40 \%)$ & $2(20 \%)$ & $1(10 \%)$ \\
\hline 2 & $3(100 \%)$ & $2(67 \%)$ & $1(33 \%)$ & $2(67 \%)$ & $0(0 \%)$ \\
\hline 3 & $0(0 \%)$ & $1(100 \%)$ & $1(100 \%)$ & $0(0 \%)$ & $1(100 \%)$ \\
\hline 5 & $1(100 \%)$ & $1(100 \%)$ & $0(0 \%)$ & $1(100 \%)$ & $1(100 \%)$ \\
\hline 8 & $1(50 \%)$ & $2(100 \%)$ & $0(0 \%)$ & $1(50 \%)$ & $2(100 \%)$ \\
\hline Total & $12(70 \%)$ & $15(88 \%)$ & $6(35 \%)$ & $6(35 \%)$ & $5(29 \%)$ \\
\hline$p$ (Fisher) & 0,36 & 0,66 & 0,66 & 0,28 & $0,01^{\star}$ \\
\hline
\end{tabular}


Choques apropriados e troca de CDI

Não observamos associação estatisticamente significante entre a troca de CDI e choques apropriados, ou seja, a proporção não foi diferente entre os pacientes que trocaram e os que não trocaram o CDI. Entretanto, as proporções de troca de CDI foram muito mais freqüentes nos pacientes que não receberam choques apropriados (figura 8).

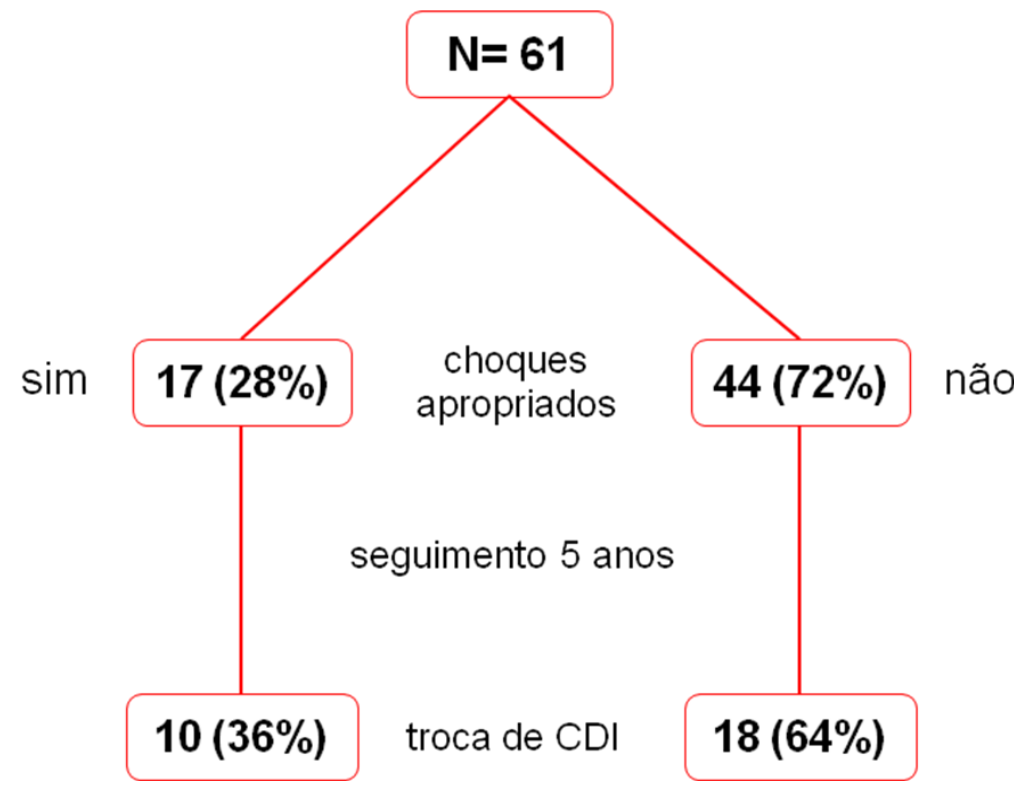

Figura 8 - Taxa de choque apropriado e trocas de CDI

\section{Medicações}

Cinquenta e dois pacientes (85\%) estavam em uso de betabloqueadores, 32 (52\%) de amiodarona e 22 (36\%) de verapamil, estes não tiveram qualquer efeito nos pacientes que receberam choques apropriados, ou seja, a proporção destes não foi diferente entre os que recebiam medicação e os que não recebiam, quer tenham indicação de prevenção primária ou secundária. 


\section{Complicações}

Houve complicações em 29 (48\%) pacientes, sendo em 25 (41\%) relacionadas ao sistema (gerador e cabo-eletrodo) e em 8 (13\%) ao procedimento cirúrgico.

As complicações do sistema foram: choque inapropriado em 22/61 (36\%) e em um destes houve necessidade de reposicionamento do eletrodo; fratura do eletrodo em $7 / 61(11 \%), 5$ desses tiveram choque inapropriado; gerador com defeito e necessidade de troca em 1/61 (2\%).

\section{Choques inapropriados}

Foram observados em $22 / 61$ pacientes (36\%) e o primeiro choque ocorreu em média $23(3-87)$ meses após o implante do CDI (percentil $25=4$ meses e percentil $75=42$ meses). Dezessete pacientes $(28 \%)$ receberam 1 choque; $2(3 \%) 2 ; 1(2 \%) 5 ; 1(2 \%) 10$ e 1 (2\%) 13; o número de choques inapropriados, além de ter sido muito mais freqüente em relação aos choques apropriados, foi em número maior por paciente.

Houve associação estatisticamente significante entre a presença do choque inapropriado e sexo masculino ( $p=0,05$ Pearson), com presença de TVNS ( $p=0,03$ Pearson), SIV >30mm ( $p=0,05$ Pearson) e uso de amiodarona $(p=0,001$ Pearson).

Ao analisar os choques inapropriados e a troca de CDI observamos associação estatisticamente significante entre ambos; a proporção de choques inapropriados foi maior entre os pacientes que trocaram CDI (54\%); já nos que não trocaram a proporção de choques foi de $21 \%$, ou seja, a chance de choque inapropriado foi mais de 4 vezes maior nos que trocaram o $\operatorname{CDI}(O R=4,3$; 
IC $=95 \%)$. Quando avaliamos os grupos de pacientes segundo a indicação observamos que as diferentes proporções de choques inapropriados entre os que trocaram e os que não trocaram o CDI deve-se ao grupo de prevenção primária, pois no grupo de prevenção secundária não tivemos evidências de efeito da troca de CDI nos choques inapropriados (tabelas 4 e 5 ).

Tabela 4 - Choques inapropriados e troca de CDI

\begin{tabular}{|c|c|c|c|c|}
\hline \multirow[b]{2}{*}{ Troca CDI } & & \multicolumn{2}{|c|}{ Choques inapropriados } & \multirow[b]{2}{*}{ Total } \\
\hline & & Não & Sim & \\
\hline \multirow{3}{*}{ Não } & $\mathbf{n}$ & 26 & 7 & 33 \\
\hline & $\%$ troca CDI & 79 & 21 & \\
\hline & $\%$ choques & 67 & 32 & \\
\hline \multirow{3}{*}{ Sim } & n & 13 & 15 & 28 \\
\hline & $\%$ troca CDI & 46 & 54 & \\
\hline & $\%$ choques & 33 & 68 & \\
\hline Total & $n$ & 39 & 22 & 61 \\
\hline$p$ (Pearson) & 0,009 & & & \\
\hline OR (IC 95\%) & $4,3(1,4-13,1)$ & & & \\
\hline
\end{tabular}

Tabela 5 - Choques inapropriados e CDI na prevenção primária

\begin{tabular}{l|c|c|c|c}
\hline \multicolumn{2}{c|}{} & \multicolumn{2}{c|}{ Choques inapropriados } & \\
\hline Troca CDI & & Não & Sim & Total \\
\hline \multirow{4}{*}{ Não } & $\mathrm{n}$ & 21 & 6 & 27 \\
\cline { 2 - 5 } & $\%$ troca CDI & 78 & 22 & \\
\cline { 2 - 5 } & $\%$ choques & 72 & 35 & \\
\hline \multirow{3}{*}{ Sim } & $\mathrm{n}$ & 8 & 11 & 19 \\
\cline { 2 - 5 } & $\%$ troca CDI & 42 & 58 & \\
\cline { 2 - 5 } & $\%$ choques & 28 & 65 & 46 \\
\hline Total & $\mathrm{n}$ & 29 & 17 & \\
\hline p (Pearson) & 0,01 & & & \\
\hline OR (IC 95\%) & $4,8(1,3-17,4)$ & & & \\
\hline
\end{tabular}


As complicações do procedimento foram: endocardite infecciosa em 2/61 (3\%); infecção do local de implante 2/61 (3\%), um deles retirou o gerador e não recolocou - tinha colocado para prevenção primária; pneumotórax 1/61 (2\%); celulite do local de implante 1/61 (2\%); hematoma no local de implante 1/61 (2\%) e retração do subcutâneo no local de implante 1/61 (2\%).

A análise estatística não mostrou associação significante entre a proporção de choques apropriados e as complicações, nem com a proporção desta e os pacientes com indicação de prevenção primária ou secundária de CDI.

\section{Sobrevida}

Ao final do estudo com 61 pacientes, 55 (90\%) estavam vivos. Seis pacientes $(10 \%)$ faleceram $(5-8 \%$ relacionados à $\mathrm{CH}, 2$ deles com choques apropriados, 1 com morte súbita; 3 sem choques apropriados, 2 deles com morte súbita) (tabela 6). 0 tempo médio até o óbito foi de 3,5 anos ( 1 - 6).

Em relação aos pacientes que foram a óbito notamos associação estatisticamente significante com a faixa etária $(p=0,02$ Fisher) e com a presença de TVNS ( $p=0,04$ Fisher).

Tabela 6 - Características clínicas e laboratoriais dos pacientes que faleceram

\begin{tabular}{|c|c|c|c|c|c|c|c|c|c|c|c|}
\hline Caso & Sexo & $\begin{array}{l}\text { Idade } \\
\text { anos }\end{array}$ & Indicação & MSF & Síncope & $\begin{array}{l}\text { SIV } \\
\mathrm{mm}\end{array}$ & TVNS & $\begin{array}{c}\text { Tempo CDI } \\
\text { (meses) }\end{array}$ & $\begin{array}{c}\text { Choque } \\
\text { apropriado }\end{array}$ & $\begin{array}{c}\text { Choque } \\
\text { inapropriado }\end{array}$ & $\begin{array}{c}\text { Causa } \\
\text { óbito }\end{array}$ \\
\hline JAHF & $\mathrm{F}$ & 13 & 1 & Não & Sim & 31 & Sim & 13 & Não & Sim & IC \\
\hline MHCR & $\mathrm{F}$ & 57 & 2 & Não & Sim & 28 & Sim & 40 & Sim & Não & TE \\
\hline MJS & $M$ & 27 & 1 & Sim & Não & 20 & Não & 32 & Não & Não & MS \\
\hline RSC & $\mathrm{F}$ & 17 & 1 & Sim & Sim & 18 & Sim & 39 & Sim & Não & MS \\
\hline RBS & $\mathrm{F}$ & 29 & 1 & Sim & Sim & 29 & Sim & 64 & Não & Sim & MS \\
\hline
\end{tabular}


Nas figuras 9 e 10 são mostradas as curvas de sobrevida do grupo todo e pela indicação. Nesta última não observamos diferença estatisticamente significante entre os grupos de prevenção primária ou secundária ( $p=0,62$ logrank).

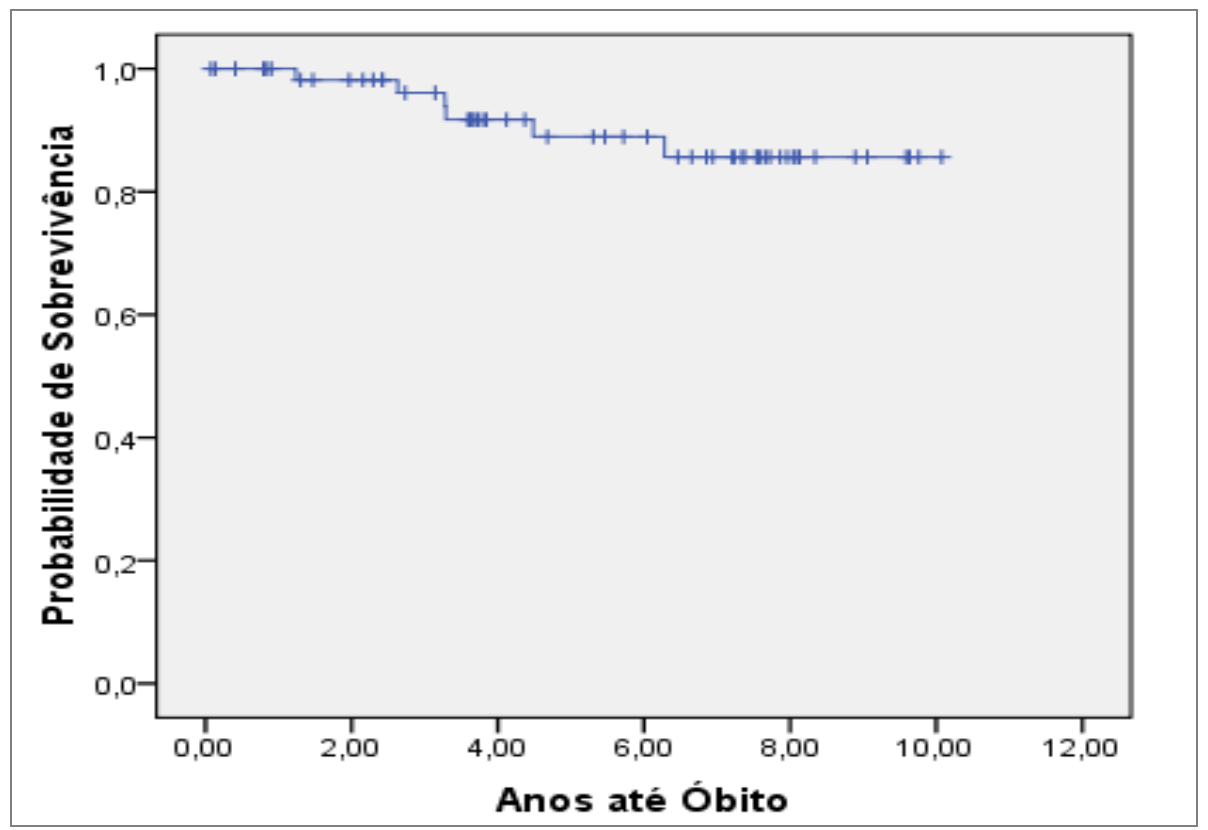

Figura 9 - Curva de sobrevida total (Kaplan-Meier) 


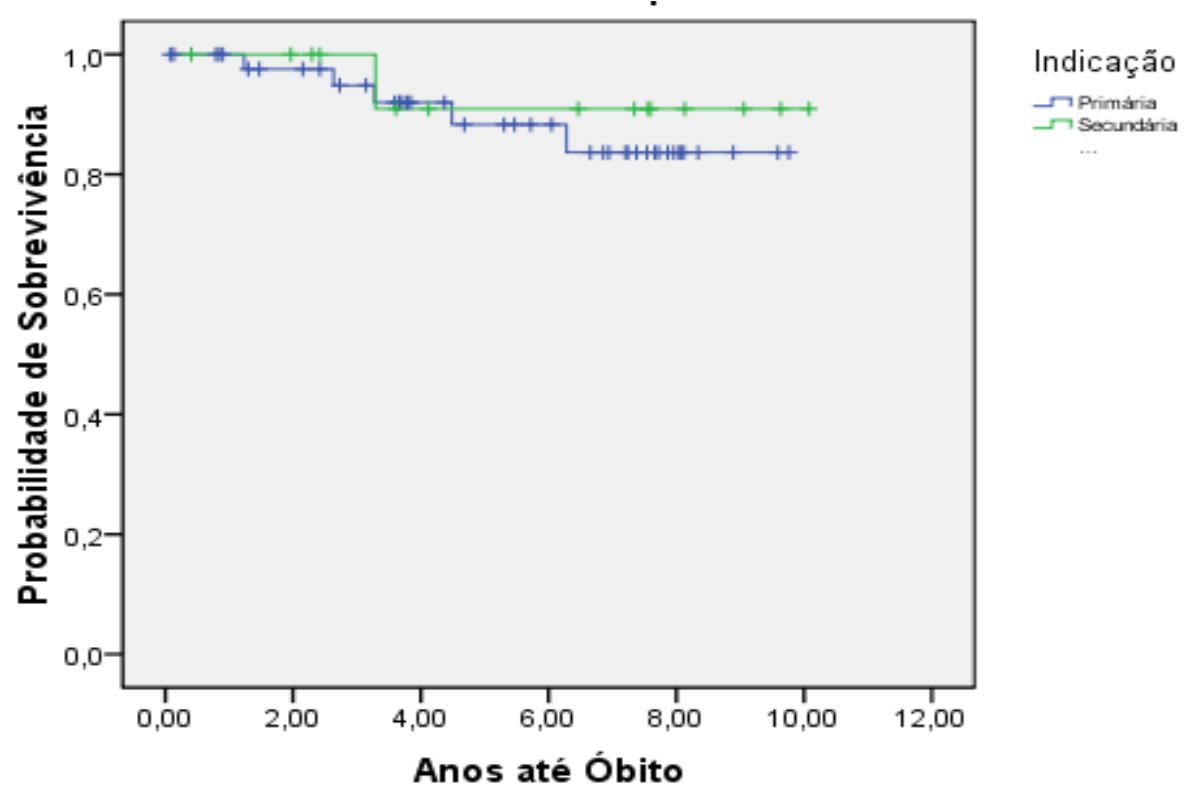

Figura 10 - Curva de sobrevida por indicação (Kaplan-Meier); ( $p=0,62$ log-rank) 
DISCUSSÃO 
Discriminar clinicamente quais os pacientes que têm maior risco de morte súbita e indicar o implante de CDI para assim melhorar a sobrevida é hoje o nosso maior desafio. Nosso estudo é o primeiro a ser realizado no Brasil com o objetivo de avaliar as características dos pacientes, as indicações, a incidência de choques apropriados, as relações entre estas e os fatores de risco, a sobrevida e óbitos, assim como as complicações. É o sétimo trabalho na literatura com casuística com mais de 45 pacientes; desses, três são multicêntricos $18,34,38$ e o que tem maior casuística foi realizado em 42 centros de referência de três continentes ${ }^{18}$.

Nossa casuística é jovem, distribuída em ambos sexos, a maioria (84\%) pouco sintomática em classes funcionais I e II, com hipertrofia septal moderada (24 mm), átrio esquerdo dilatado (45 mm) e função sistólica preservada, que a faz comparável à da literatura 18,34,36,38,40,41 (tabelas 1 e 7). 0 tempo de seguimento médio (5 anos) é dos maiores e só comparável ao de Begley et al. ${ }^{36 .}$

A indicação de CDI em $75 \%$ dos pacientes foi como prevenção primária e em $25 \%$ secundária, semelhante ao observado na literatura $18,34,36,38,40,41$ (média de $72 \pm 9 \%$ na primária), ou seja, há um excesso de indicação com o intuito de salvar vidas e, em nossa opinião, com resultado aquém da expectativa. Ao analisar associação entre a indicação e as variáveis demográficas, clínicas e exames complementares encontramos maior proporção de história familiar de morte súbita no grupo de prevenção primária ( $p=0,007$ Fisher); maior proporção de taquiarritmias ventriculares no grupo de prevenção secundária ( $p=<0,0001$ Fisher), e neste grupo a proporção de pacientes que faziam uso de amiodarona também era maior ( $p=0,01$ Pearson). 


\section{Tratamento}

O CDI mostrou-se eficaz no controle das taquiarritmias em $17(28 \%)$ de nossos pacientes com média de 2 choques apropriados por paciente, o que está próximo do observado por Syska et al. ${ }^{41}$ que estudaram um grupo de 104 pacientes e encontraram $26 \%$ de choques apropriados, porém acima do observado por Maron et al. 18, 34 (20\%-23\%), Begley et al. ${ }^{36}(20 \%)$, Marin et al. ${ }^{38}$ $(22 \%)$ e Lin et al. 40 (16\%). Entretanto, isto é muito pouco, considerando tratarse de pacientes tidos como de alto risco. Ao analisar a proporção de choques apropriados por ano observamos que foi de $7 \%$ em nossa casuística, semelhante à observada na literatura ${ }^{34,36}$ e próxima à de Syska et al. ${ }^{41}$ que foi $5.6 \%$ (tabela 7$)$. 


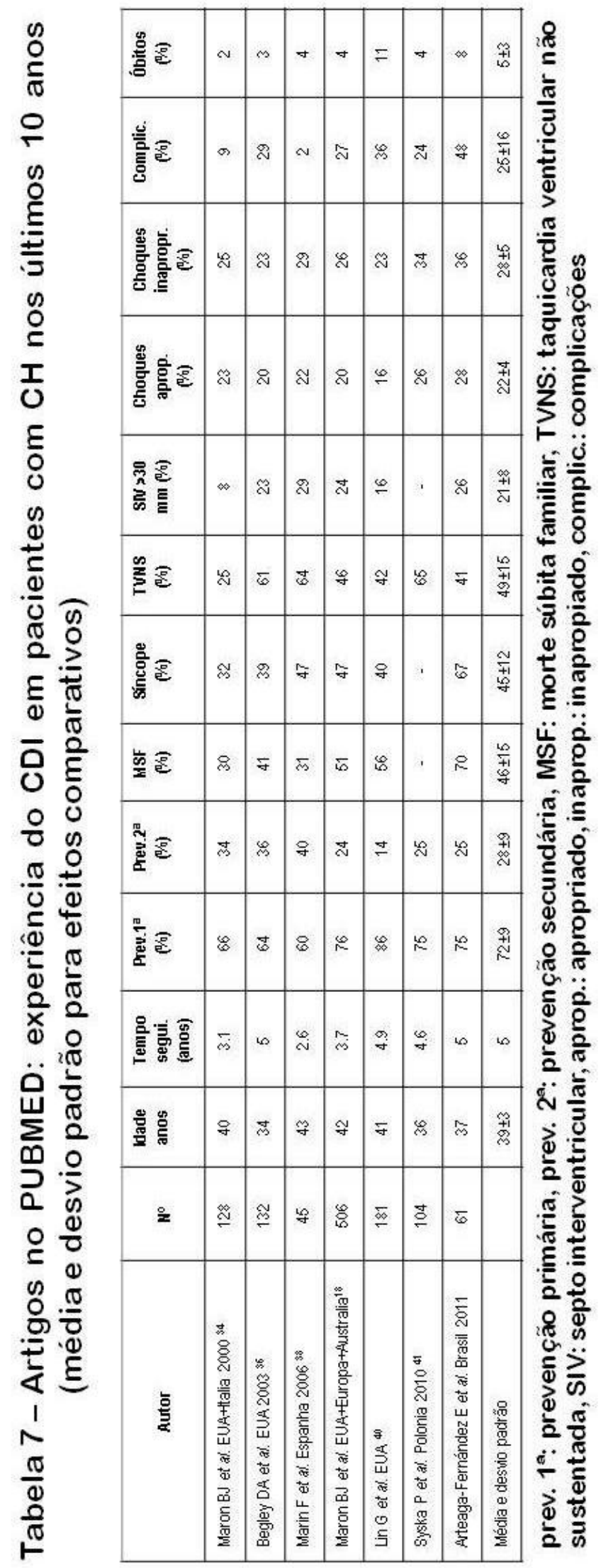


Numa estimativa de 100 implantes de CDI teríamos 6 choques apropriados no grupo de prevenção primária e 7 no grupo de prevenção secundária no primeiro ano, próximo ao observado por Syska et al. ${ }^{41}$ (4 e 8), porém diferente de Maron et al. ${ }^{18}$ (4 e 11) e de Marin et al. ${ }^{38}$ (2 e 11).

É possível que essas diferenças decorram do fato da doença ser resultado de mais de 900 mutações o que, em teoria, faz com que cada paciente seja diferente do outro. Isto é corroborado pelo fato de as maiores casuísticas serem de estudos multicêntricos com indivíduos de diferentes etnias. Em nosso estudo temos menor número de pacientes, mas a casuística tem origem multiracial e com muita miscigenação.

Os choques apropriados ocorreram longo tempo após a colocação do CDI, o que já é descrito na literatura $18,36,41$. Não observamos associação estatisticamente significante entre choques apropriados e troca do CDI e, paradoxalmente, a proporção de troca de CDI foi mais frequente no grupo de pacientes que não receberam choques apropriados (64\% vs. $36 \%)$.

Choques apropriados foram observados em maior proporção nas faixas etárias abaixo de 25 e acima de 40 anos, semelhante ao observado por Begley et al. 36; entretanto, não observamos diferença estatisticamente significante entre elas. Para Maron et al. ${ }^{18}, 40 \%$ dos pacientes que tiveram choques apropriados estavam com menos de 40 anos. Isto está de acordo com a observação de que indivíduos jovens apresentam maior risco de MSC 1, 5, 12, 13 .

A análise de associação entre os fatores de risco e choques apropriados mostrou que apenas a síncope foi estatisticamente significante $(p=0,03$ Pearson); quando analisamos associação entre choques apropriados e o número de fatores de risco por paciente não observamos significância estatística; quando agrupamos os pacientes com menos de 2 ou com mais de 2 
fatores de risco, observamos diferença estatisticamente significante $(p=0,05$ Fisher); entretanto, quando analisamos associação entre choques apropriados, número de fatores de risco por paciente e indicação, não observamos significância estatística, mesmo quando agrupamos com menos e mais do que 2 fatores de risco. Já, quando avaliamos a associação entre o número de choques apropriados e fatores de risco observamos associação estatisticamente significante ( $p=0,01$ Fisher) com taquiarritmias ventriculares, que ocorreram no grupo de prevenção secundária.

Begley et al. 36 num estudo com 132 pacientes, não observaram nenhuma relação entre choques apropriados e fatores de risco; Marin et al. ${ }^{38}$, em estudo multicêntrico com 45 pacientes, observaram associação entre choques apropriados e história de parada cardíaca recuperada ou TVS; já Maron et al. ${ }^{18}$, no maior estudo multicêntrico com mais de 500 pacientes de três continentes, não observaram diferença no número de choques apropriados entre 1, 2, 3 ou 4 fatores de risco; Syska et al. ${ }^{41}$ observaram que a presença de TVNS foi o único fator de risco preditivo de choques apropriados no grupo de prevenção primária (valor preditivo positivo $22 \%$, negativo $96 \%$, sensibilidade $92 \%$ e especificidade $35 \%$ ).

Com este panorama atual, principalmente pelas características inerentes à doença, temos ainda muito a aprender e conhecer. É possível que, em futuro próximo, a biologia molecular através da genética, também contribua para o conhecimento que nos ajudará a melhor identificar o subgrupo de alto risco, evitando-se mortes com CDI, objetivo principal da nossa busca. 


\section{Complicações}

Nosso índice de complicações foi muito elevado 29/61 (48\%), quase metade dos pacientes tiveram complicações; destas, $25 / 29$ (86\%) devido a falhas do sistema gerador e cabo-eletrodo. Na literatura 18, 34, 36, 38, 40, 41 a média é de $25 \pm 16 \%$ (tabela 7 ).

Os choques inapropriados foram observados em $22 / 61$ (36\%) dos pacientes; estes foram muito mais frequentes em relação aos choques apropriados e em maior número por paciente (um chegou a receber 13 choques inapropriados) o que configura, em nossa opinião, o principal problema do CDI. Na literatura 18, 34, 36, 38, 40, 41 choque inapropriado é observado em média em $28 \pm 5 \%$ dos pacientes e a nossa foi a maior de todas. Observamos em nosso grupo associação estatisticamente significante entre os choques inapropriados e o sexo masculino ( $p=0,05$ Pearson), idade menor que 35 anos ( $p=0,05$ Fisher), TVNS ( $p=0,03$ Pearson), SIV >30 mm ( $p=0,05$ Pearson) e uso de amiodarona ( $p=0,001$ Pearson). Estes pacientes tiveram maior proporção de troca de CDI, principalmente os que colocaram como prevenção primária e chance quatro vezes maior de receber choques inapropriados (OR= 4,3 IC $95 \%)$.

As causas de choques inapropriados pelo CDI são taquicardia sinusal, fibrilação atrial, oversensing da onda $T$, fratura do cabo-eletrodo e mau funcionamento do dispositivo. Lin et al. ${ }^{40}$, em estudo retrospectivo de 181 pacientes, observaram que os choques inapropriados e complicações do sistema são elevados e mais comuns em pacientes jovens e com fibrilação atrial associada. Assim, a idade $<35$ anos ao implante de CDI é preditiva de choque inapropriado no futuro $(p<0,01)$. 
As complicações decorrentes do procedimento cirúrgico também foram elevadas $8 / 61(13 \%)$, resultando em endocardite infecciosa, infecção local, hematoma e pneumotórax. Para outros autores esse índice de complicações é menor que $5 \% 18,36,40,41$.

\section{Óbitos e sobrevida}

Ao final do seguimento ocorreram $5 / 61(8 \%)$ óbitos relacionados à $\mathrm{CH}$.

Como era de se esperar, a maioria (60\%) faleceu de morte súbita, $80 \%$ era do sexo feminino com menos de 30 anos, referia síncope, apresentava TVNS e tinha colocado CDI para prevenção primária; $60 \%$ dos pacientes receberam choque apropriado e $40 \%$ tiveram choques inapropriados (tabela 7 ).

A sobrevida foi de $90 \%$, e não houve diferença estatisticamente significante entre o grupo que teve indicação primária ou secundária.

Podemos concluir que temos um tratamento apropriado para evitar a morte súbita que é a mais temida complicação da cardiomiopatia hipertrófica, que esta ocorre em um subgrupo pequeno e que todos os fatores de risco que conhecemos têm baixo poder preditivo. Portanto, mesmo que utilizemos um ou vários dos fatores de risco conhecidos não conseguimos alta sensibilidade e especificidade. Por outro lado, o cardiodesfibrilador é eficaz, mas trazendo em si alto índice de complicações com resultados inaceitáveis. De fato, estas são em número maior do que os choques apropriadas (1:2 - 1:4), sem contar ainda as complicações importantes decorrentes do procedimento cirúrgico. Hão que ser incluídas no rol das complicações as de origem emocional, que afetam a todos, mais frequentes nos jovens, determinando quadros de depressão. 
Como não seria possível planejar um estudo duplo-cego, pois o que está em jogo é a vida, seria o momento de repensar o que fizemos até agora e propor novas diretrizes com todas as sociedades de cardiologia envolvidas e planejar um estudo multicêntrico para chegarmos a um consenso e ir ao encontro de nosso anseio, consubstanciado em buscar a diminuição dos índices de morte súbita dos portadores desta doença de causa genética, sem esperança de cura.

\section{Limitações do estudo}

As limitações podem ser explicadas pelo fato da doença ser infrequente, a taxa de morte súbita baixa, o que determina longo tempo de estudo, e o alto valor do CDI - que limita sua indicação, uma vez que é custeado pelos cofres públicos.

Nossa casuística, apesar de pequena, representa $10 \%$ do maior trabalho multicêntrico já publicado e com a maior casuística (mais de $\mathbf{5 0 0}$ pacientes recrutados em 42 centros de três continentes). É um estudo prospectivo de um único centro de referência terciário e com a mesma equipe que diagnostica, trata e acompanha os pacientes. 
CONCLUSÕES 
1. Cardiodesfibrilador implantável conseguiu prevenir a morte cardíaca de 21/61 (28\%) pacientes portadores de cardiomiopatia hipertrófica, na proporção de 7 pacientes por ano.

2. As proporções de choques apropriados no $1^{\circ}$ ano foram de $6 \%$ na indicação para prevenção primária e 7\% na prevenção secundária; numa estimativa, no primeiro ano, de 100 implantes de cardiodesfibriladores, 6 vidas foram salvas na indicação de prevenção primária e 7 na prevenção secundária.

3. Dos quatro fatores de risco convencionais, apenas a história de morte súbita familiar teve associação estatisticamente significante com a indicação de prevenção primária.

4. Os choques apropriados tiveram associação estatisticamente significante com a síncope e com a presença de mais de dois fatores de risco.

5. Incidência de complicações foi observada em quase metade dos pacientes, sendo mais frequente àquelas relacionadas ao sistema gerador e cabo-eletrodo, e em proporção bem menor às relacionadas ao procedimento cirúrgico. Os choques inapropriados ocorreram em mais de 1/3 dos pacientes e tiveram associação estatisticamente significante com o sexo masculino, septo interventricular $>30 \mathrm{~mm}$, presença de taquicardia ventricular não sustentada, uso de amiodarona e com a troca de cardiodesfibrilador. 


\section{APROVAÇÃO}

A Comissão de Ética para Análise de Projetos de Pesquisa CAPPesa da Diretoria Clínica do Hospital das Clínicas e da Faculdade de Medicina da Universidade de São Paulo, em sessão de 25/08/2010, APROVOU O Protocolo de Pesquisa n 0383/10, intitulado: "AVALIAÇĀO DA EFICÁCIA DO CARDIOVERSOR-DESFIBRILADOR IMPLANTÁVEL NA PREVENÇĀO DE MORTE SÚBITA EM PACIENTES COM CARDIOMIOPATIA HIPERTRÓFICA" apresentado pela COMISSÃO CIENTíFICA DO INCOR, inclusive O Termo de Consentimento Livre e Esclarecido versão 1 de 27.04.10.

Cabe ao pesquisador elaborar e apresentar à CAPPesq, os relatórios parciais e final sobre a pesquisa (Resolução do Conselho Nacional de Saúde n 196, de 10/10/1996, inciso IX.2, letra "c").

Pesquisador (a) Responsável: Edmundo Arteaga-Fernández

Pesquisador (a) Executante: Paulo de Tarso Jorge de Medeiros

CAPPesq, 26 de Agosto de 2010

Prof. Dr. Eduardo Massad Presidente da Comissão de Ética para Análise de Projetos de Pesquisa

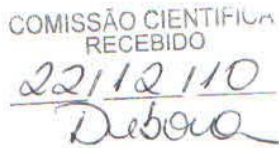

Comissão de Ética para Análise de Projetos de Pesquisa do HCFMUSP e da FMUSP Diretoria Clínica do Hospital das Clínicas da Faculdade de Medicina da Universidade de Sāo Paulo Rua Ovídio Pires de Campos, 225, $5^{\circ}$ andar - CEP 05403010 - São Paulo - SP Fone: C11 30696442 Fax: 01130696492 e-mail: cappesq@hcnet.usp.br/ secretariacappesq2@hcnet.usp.br 
REFERÊNCIAS 
1. Teare D. Asymmetrical hypertrophy of the heart in young adults. $\mathrm{Br}$ Heart J. 1958;20:1-8.

2. Braunwald E, Lambrew C, Rockoff D, Ross J Jr, Morrow AG. Idiopathic hypertrophic subaortic stenosis. I: A description of the disease based upon an analysis of 64 patients. Circulation. 1964;30(Suppl. IV):1-217.

3. Maron BJ, Bonow RO, Cannon III RO, Leon MB, Epstein SE. Hypertrophic cardiomyopathy: Interrelations of clinical manifestations, pathophysiology, and therapy (two parts). N Engl J Med. 316(13):780-9, 1987;316(14):844-52.

4. Maron BJ. Hypertrophic cardiomyopathy: A systematic review. JAMA. 2002;287:1308-20.

5. Maron BJ, McKenna, WJ, Danielson GK, Kappenberger LJ, Kuhn HJ, Seidman CE, Shah PM, Spencer WH, Spirito P, Ten Cate Wigle ED. American College of Cardiology/European Society of Cardiology clinical expert consensus document on hypertrophic cardiomyopathy. A report of the American College of Cardiology Foundation Task Force on Clinical Expert Consensus Documents and the European Society of Cardiology Committee for Practice Guidelines. J Am Coll Cardiol. 2003;42:1687-713.

6. Watkins $\mathrm{H}$. Multiple disease gene cause hypertrophic cardiomyopathy. $\mathrm{Br}$ Heart J. 1994;72(Suppl.):S4-S9.

7. Ho CY. Genetics and Clinical Destiny: Improving Care in Hypertrophic Cardiomyopathy. Circulation. 2010;122;2430-40.

8. Maron BJ, Gardin JM, Flack JM, Gidding SS, Kurosaki TT, Bild DE. Prevalence of hypertrophic cardiomyopathy in a general population of young adults. Echocardiograpic analysis of 4111 subjects in the CARDIA Study. Coronary Artery Risk Development in (Young) Adults. Circulation. 1995; 92(4):785-9.

9. Hugues SE. The pathology of hypertrophic cardiomyopahty. Histopathology. 2004;44:412-27. 
10. Cecchi F, Olivotto I, Gistri R, Lorenzoni R, Chiriatti G, Camici PG. Coronary microvascular dysfunction and prognosis in hypertrophic cardiomyopathy. N Engl J Med. 2003;349:1027-35.

11. Tsoutsman T, Lam L, Semsarian C. Genes, calcium and modifying factors in hypertrophic cardiomyopathy. Clin Exp Pharmacol Physiol. 2006;33:139-45.

12. Elliott PM, Poloniecki J, Dickie S, Sharma S, Monserrat L, Varnava A, Mahon NG, McKenna WJ. Sudden death in hypertrophic cardiomyopathy: identification of high risk patients. J Am Coll Cardiol. 2000;36:2212-8.

13. Maron BJ, Olivotto I, Spirito P, Casey SA, Bellone P, Gohman TG, Graham KJ, Burton DA, Cecchi F. Epidemiology of hypertrophic cardiomyopathyrelated death: revisited in a large non-referral-based patient population. Circulation. 2000;102:858-64.

14. McKenna WJ, Oakley CM, Krikler DM, Goodwing JF. Improved survival with amiodarone in patients with hypertrophic cardiomyopathy and ventricular tachycardia. Br Heart J. 1985;53:412-416.

15. Araujo AQ, Arteaga E, lanni BM, Buck PC, Rabello R, Mady C. Effect of Losartan on left ventricular diastolic function in patients with nonobstructive hypertrophic cardiomyopathy. Am J Cardiol. 2005;96:1563-7.

16. Melacini P, Maron BJ, Bobbo F, Basso C, Tokajuk B, Zucchetto M, Thiene G, lliceto S. Evidence that pharmacological strategies lack efficacy for the prevention of sudden death in hypertrophic cardiomyopathy. Heart. 2007;93:708-10.

17. Cuoco FA, Spencer WH, Fernandes VL, Nielsen CD, Nagueh S, Sturdivant JL, Leman RB, Wharton JM, Gold MR. Implantable cardioverterdefibrillator theraphy for primary prevention of sudden death after alcohol septal ablation of hypertrophic cardiomyopathy. J Am Coll Cardiol. 2008;52:1718-23. 
18. Maron BJ, Spirito P, Shen WK, Haas TS, Formisano F, Link MS, Epstein AE, Almquist AK, Daubert JP, Lawrenz T, Estes NAM, Favale S, Piccininno M, Winters SL, Betocchi S, Arribas F, Sherrid MV, Buja G, Semsariam C, Bruzzi P. Implantable cardioverter-defibrillators and prevention of sudden cardiac death in hypertrophic cardiomyopathy. JAMA. 2007;298:405-12.

19. Dimitrow PP, Chojnowska L, Rudzinski T, Piotrowsky W, Ziótkowska L, Wojtarowicz A, Wycisk A, Dabrowska-Kugacka A, Nowalany-Kozielska E, Sobkowicz B, Wróbel W, Aleszewicz-Baranowska J, Rynkiewicz A, LobozGrudzien K, Marchel M, Wysokinsky A. Sudden death in hypertrophic cardiomyopathy: old risk factors re-assessed in a new modelo os maximalized follow-up. Eur Heart J. 2010;31:3084-93.

20. Spirito P, Autore C, Rapezzi C, Bernabò P, Badagliacca R, Maron MS, Bongioanni S, Coccolo F, Estes NAM, Barillà CS, Biagini E, Quarta G, Conte MR, Bruzzi P, Maron B. Syncope and risk of sudden death in hypertrophic cardiomyopathy. Circulation. 2009;119:1703-10.

21. Spirito $P$, Bellone $P$, Harris KM, Bernabó $P$, Bruzzi P, Maron B. Magnitude of left ventricular hypertrophy and risk of sudden death in hypertophic cardiomyopathy. N Engl J Med. 2000;342:1778-85.

22. Elliott PM, Blanes JRG, Mahon NG, Poloniecki JD, McKenna WJ. Relation between severity of left-ventricular hypertrophy and prognosis in patients with hypertrophic cardiomyopathy. Lancet. 2001;357:420-4.

23. Olivotto I, Maron MS, Autore C, Lesser JR, Rega L, Casolo G, De Santis M, Quarta G, Nistri S, Cecchi F, Salton CJ, Udelson JE, Manning WJ, Maron BJ. Assessment and significance of left ventricular mass by cardiovascular magnetic resonance in hypertrophic cardiomyopathy. $J$ Am Coll Cardiol. 2008;52:559-66.

24. Monserrat L, Elliott PM, Gimeno JR, Sharma S, Penas-Lado M, McKenna WJ. Non-sustained ventricular tachycardia in hypertrophic cardiomyopathy: an independent marker of sudden death risk in young patients J Am Coll Cardiol. 2003;42:873-9. 
25. Adabag AS, Casey SA, Kuskowski MA, Zenovich AG, Maron BJ. Spectrum and prognostic significance of arrhythmias on ambulatory Holter electrocardiogram in hypertrophic cardiomyopathy. J Am Coll Cardiol. 2005;45:697-704.

26. O'Hanlon R, Grasso A, Roughton M, Moon JC, Clark S, Wage R, Webb J, Kulkarni M, Dawson D, Sulaibeekh L, Chandrasekaran B, Bucciarelli-Ducci C, Pasquale F, Cowie MR, McKenna WJ, Sheppard MN, Elliott P, Pennell DJ, Prasad SK. Prognostic significance of myocardial fibrosis in hypertrophic cardiomyopathy. J Am Coll Cardiol 2010;56:867-74.

27. Shiozaki AA, Senra T, Arteaga E, Pita CG, Martinelli Filho M, Avila LF, Parga Filho JR, Mady C, Rochitte CE. Myocardial fibrosis in patients with hypertrophic cardiomyopathy and high risk for sudden death. Arq Bras Cardiol. 2010;94:535-40.

28. Arteaga E, Araújo $A Q$, Bernstein M, Ramires FJ, lanni BM, Fernandes $F$, Mady C. Prognostic value of the collagen volume fraction in hypertrophic cardiomyopathy. Arq Bras Cardiol. 2009;92:210-4.

29. Harris KM, Spirito P, Maron MS, Zenovich AG, Formisano F, Lesser JR, Mackey-Bojack S, Manning WJ, Udelson JE, Maron BJ. Prevalence, clinical profile, and significance of left ventricular remodeling in the endstage phase of hypertrophic cardiomyopathy. Circulation 2006;114:21625.

30. Maron MS, Finley JJ, Bos JM, Hauser TH, Manning WJ, Haas TS, Lesser JR, Udelson JE, Ackerman MJ, Maron BJ. Prevalence, clinical significance and natural history of left ventricular apical aneurysms in hypertrophic cardiomyopathy. Circulation 2008;118:1541-9.

31. Elliott PM, Gimeno JR, Tomé MT, Shah J, Ward D, Thaman R, Mogensen J, McKenna WJ. Left ventricular outflow tract obstruction and sudden death risk in patients with hypertrophic cardiomyopathy. Eur Heart $J$ 2006;27:1933-41. 
32. Noseworthy PA, Rosenberg MA, Fifer MA, Palacios IF, Lowry PA, Ruskin JN, Sanborn DM, Picard MH, Vlahakes GJ, Mela T, Das S. Ventricular arrhythmia following alcohol ablation for obstructive hypertrophic cardiomyopathy. Am J Cardiol 2009;104:128-32.

33. Mirowski M, Reid PR, Mower MM, Watkins L, Gott VL, Schauble JF, Langer A, Heilman MS, Kolenik SA, Fischell RE, Weisfeldt ML. Termination of malignant ventricular arrhythmias with an implanted automatic defibrillator in human beings. N Engl J Med 1980;303:322-4.

34. Maron BJ, Shen WK, Link MS, Epstein AE, Almquist AK, Daubert JP, Bardy GH, Favale S, Rea RF, Boriani G, Estes M, Spirito P. Efficacy of implantable cardioverter-defibrillator for the prevention of sudden death in patients with hypertrophic cardiomyopathy. N Eng/ J Med 2000;342:365-74.

35. Epstein AE. Benefits of the implantable cardioverter-defibrillator. J Am Coll Cardiol 2008;52:1122-1127. N Engl J Med 2000;342:365-73.

36. Begley DA, Mohiddin SA, Tripodi D, Winckler JB, Fananapazir L. Efficacy of implantable cardioverter defibrillator therapy for primary and secondary prevention of sudden death in hypertrophic cardiomyopathy. PACE 2003;26:1887-1896.

37. Vieira AP, Adragão PP, Santos KR, Morgado FB, Cavaco DM, Rossi R, Abecasis M, Neves JP, Bonhosrst D, Gomes JL, Gomes RS. Implantable cardioverter-defibrillator in hypertrophic cardiomyopathy. Rev Port Cardiol 2005;24:407-15.

38. Marín F, Gimeno JR, Payá E, García-Alberola A, Pérez-Alvarez L, Fernández X, de la Morena G, Sogorb F, Castro-Beiras A, Valdés M, Martínez JG, Monserrat L. The implantable cardioverter-defibrillator and hypertrophic cardiomyopathy: experience at three centers. Rev Esp Cardiol 2006;59:537-44.

39. Woo A, Monakier D, Harris L, Hill A, Shah P, Wigle ED, Rakowsky H, Rozenblyum E, Cameron DA. Determinants of implantable defibrillator 
discharges in high-risk patients with hypertrophic cardiomyopathy. Heart 2007;93:1044-1045.

40. Syska P, Przybylski A, Chojnowska L, Lewandowski M, Sterliński M, Maciag A, Gepner K, Pytkowski M, Kowalik I, Maczyńska-Mazuruk R, Ruzyłło W, Szwed H. Implantable cardioverter-defibrillator in patients with hypertrophic cardiomyopathy: efficacy and complications of the therapy in long-term follow-up. J Cardiovasc Eletrophysio/ 2010;21:883-9.

41. Lin G, Nishimura RA, Gersh BJ, Ommen S, Ackerman M, Brady PA. Device complications and inappropriate implantable cardioverter-defibrillator shoks in patients with hypertrophic cardiomyopathy. Heart 2009;95:70914.

42. ACC/AHA/ESC 2006 Guidelines for management of patients with ventricular arrhythmias and the prevention of sudden death. Circulation 2006;114:1088-132.

43. Guidelines for the diagnosis and management of syncope (version 2009). The task force for the diagnosis and management of syncope of the European Society of Cardiology. Eur Heart J 2009;30:2631-2671.

44. Martinelli Filho M, Zimerman LI, Lorga AM, Vasconcelos JTM, Rassi Jr A. Guidelines for implantable electronic cardiac devices of the Brazilian Society of Cardiology. Arq Bras Cardio/2007;89(6):e210-e238.

45. Medeiros PTJ, Martinelli M, Arteaga E, Costa R, Siqueira S, Mady C, Piegas L, Ramires JAF. Hypertrophic cardiomyopathy: the importance of arrhythmic events in patients at risk for sudden death. Arq Bras Cardiol 2006;87:649-57.

46. Henry WL, De Maria AN, Gramiak R, King DL, Kisslo JA, Popp RL, Sahn DJ, Schiller NB, Tajik A, Feichholz LE, Weyman AE. Report of the American Society of Echocardiography Committee on Nomenclature and Standards in Two-dimensional Echocardiography. Circulation 1980;62:212-17. 
47. Teichholz LE, Kreulen T, Herman MV, Gorlin R. Problems in echocardiographic volume determinations: echocardiographicangiographic correlations in the presence or absence of asynergy. Am J Cardiol1976;37:7-11 